\title{
Interacción entre estudiantes y aprendizaje \\ en grupos pequeños *
}

\section{Noreen M. Webb**}

Universidad de California.

Traducción de Laura Pla

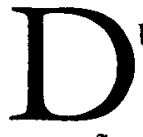

URANTE los últimos años ha aumentado el interés por la influencia que ejercen los grupos pequeños en régimen de cooperación sobre el aprendizaje. Las investigaciones sobre el aprendizaje en régimen de cooperación se han centrado en pequeños grupos de estudiantes que trabajaban en temas académicos. La característica clave que distingue las situaciones cooperativas de otras situaciones de aprendizaje es la interacción entre los estudiantes. Los estudiantes que trabajan en una situación grupal suelen hacerlo juntos para completar tareas, mientras que los estudiantes que trabajan en otras situaciones lo hacen desde sus asientos o reciben la instrucción en grupos más amplios, en los cuales la mayor parte de la interacción tiene lugar entre el profesor y el estudiante.

Mientras que varios informes han presentado imágenes favorables del aprendizaje cooperativo (Johnson, I981; Johnson y Johnson, 1974; Slavin, 1977, 1980a, I980b), no todos los informes llegan a la conclusión de que el trabajo en grupos pequeños es beneficioso para el aprendizaje. Michaels ( 1977 ) llega a la conclusión de que la competencia individual producía logros mayores que el trabajo en grupo. Incluso los fervientes defensores del aprendizaje en régimen de cooperación reconocen las discrepancias entre los descubrimientos relativos a investigacio- nes que comparan situaciones cooperativas e individuales. Además, señalan las inconsistencias entre los estudios que comparan los logros entre diferentes métodos de aprendizaje en régimen de cooperación. Las inconsistencias se han explicado en base a las características de las técnicas de aprendizaje cooperativo, a las situaciones, mediciones, diseños experimentales (Slavin, 1980a), las características de los alumnos (grupo étnico, estatus económico) y la materia a estudiar (Sharan, 1980). Sin embargo, se han investigado menos los procesos de interacción que se establecen en los grupos pequeños para ayudar a explicar los resultados inconsistentes obtenidos en los grupos en régimen de cooperación. Los diferentes modelos de interacción grupal pueden dar lugar a diferentes resultados: algunos tipos de interacción pueden resultar beneficiosos para alcanzar el éxito, mientras que otros tipos pueden ser negativos. $\mathrm{Si}$ bien la importancia de la interacción para el aprendizaje se reconoce con claridad - es a través del proceso de interacción y comunicación que se produce dentro de los grupos pequeños que cooperan en tareas académicas como estos métodos de aprendizaje por equipos intentan influir en el aprendizaje cognitivo de los alumnos" (Sharan, 1980, 242)-, pocos estudios han examinado sistemáticamente la interacción en los grupos. La mayor parte de los estudios han intentado predecir los

* "Student interaction and learning in small groups". Review of Educational Research, 1982, 52 (3), 421-449. Reproducido con autorización. (c) de esta traducción, Infancia y Aprendizaje, 1984.

** Dirección del autor. Graduate School of Education, University of California. Los Angeles, C.A. 90024. 
logros a partir de algunas características del individuo, del grupo o de la situación de trabajo. Sin datos sobre las experiencias de los estudiantes en los grupos, dichos trabajos presentan cuadros incompletos de la influencia que ejerce el trabajo en grupo sobre el aprendizaje individual.

Este trabajo presenta un resumen de las investigaciones que se han ocupado de las experiencias que tienen los estudiantes en la interacción en grupos pequeños. I.a estructura de esta revisión responde a un sistema de dos niveles, enlazando la interacción con los logros del aprendizaje y las caracteristicas de la situación de aprendizaje con la interacción. Se examina la investigación existente sobre la relación entre interacción y la investigación que explora los indicadores de la interacción en grupos pequeños. También se examinan los mecanismos que han permitido elaborar hipótesis de cómo se relaciona la interacción en los grupos con los resultados obtenidos. En la figura I se resumen las variables de la interacción, los indicadores de la interacción del grupo y los mecanismos que enlazan la interacción con los resultados. Siguiendo la presentación y evaluación de los resultados de las investigaciones, se discuten los aspectos metodológicos y sustantivos como posibles explicaciones de relaciones inconsistentes entre caracteristicas de "input", interacción y resultados, y como directrices para futuras investigaciones.

FIGLRA 1

- Variables en el sistema «input»-interacción-rendimiento

CARACTERISTICAS DEL INPUT INTERACCION RENDIMIENTO

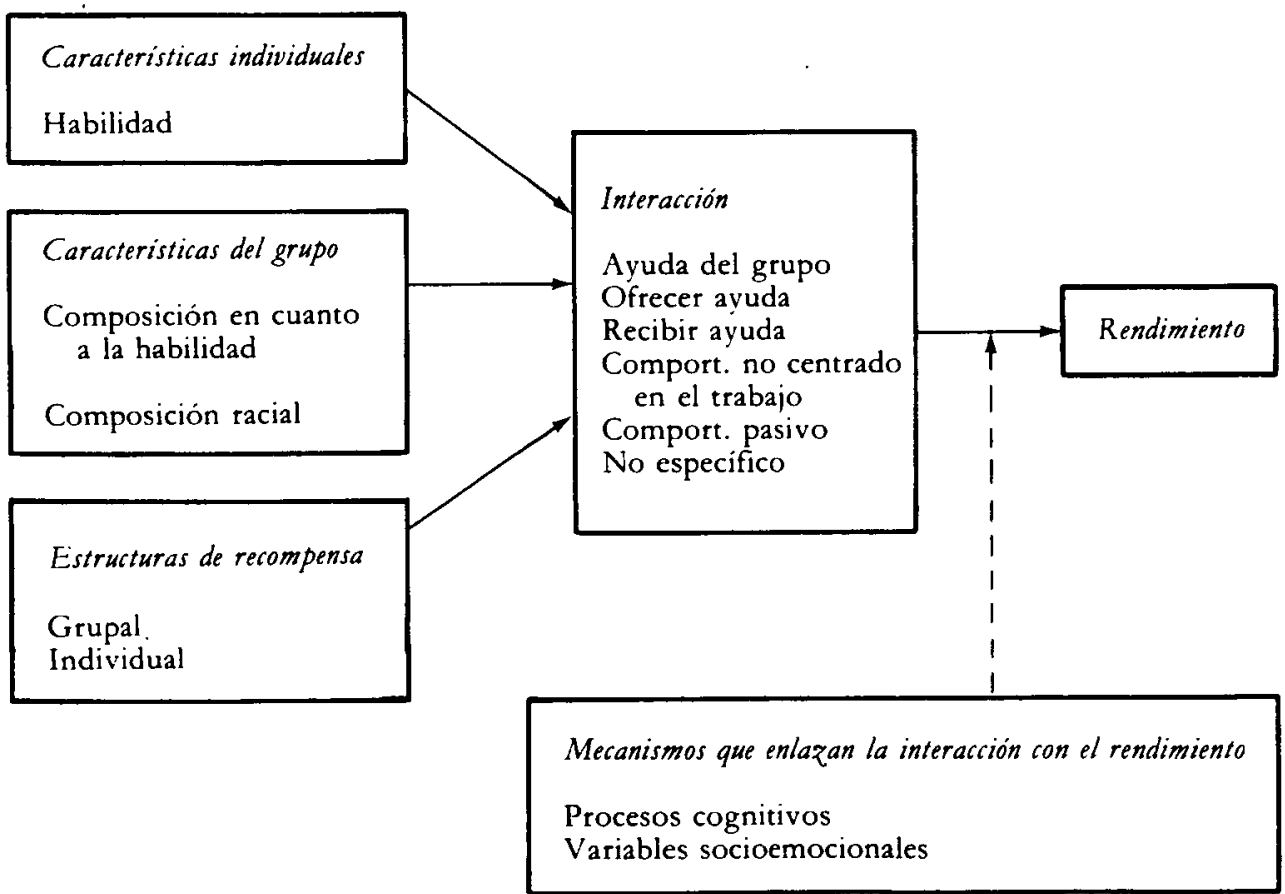

Las investigaciones a analizar fueron seleccionadas según dos criterios. En primer lugar, todas las investigaciones retenidas han utilizado material educativo para el aprendizaje en grupos pequeños. El centro de interés del trabajo en grupos que la productividad del grupo. El énfasis en las tareas educativas y en el aprendizaje elimina la mayor parte de la voluminosa bibliografía sobre dinámica de grupos. Fn segundo lugar, se han tenido en cuenta sólo aquellas investigaciones que miden de forma sistemática la inte- 
racción en grupos. Este criterio excluye los estudios que sólo aportan observaciones anecdóticas de los grupos durante el trabajo.

\section{INTERACCION Y LOGRO: RESULTADOS DE LAS INVESTIGACIONES}

\section{Comportamiento de ayuda}

La variable de interacción más corriente utilizada para predecir los resultados en los grupos pequeños es el comportamiento de ayuda. La mayor parte de los estudios han examinado la frecuencia de la ayuda en el grupo, sin diferenciar entre quienes ofrecen la ayuda y quienes. la reciben. Sólo unos pocos estudios han intentado determinar si lo que resulta beneficioso para los resultados es el hecho de ofrecer o recibir la ayuda.

Ayuda del grupo.--La evidencia de la - elación existente entre el comportamiento de ayuda del grupo y los resultados proviene de ocho estudios sobre el trabajo realizado en grupos pequeños en el aula. Dos estudios correlacionan el comportamiento de ayuda y los resultados en grupos formados por dos personas con órdenes de trabajar juntos (Hanelin, 1978; Johnson, 1979). Los otros seis estudios contrastan las condiciones de recompensa grupal e individual. En la condición de recompensa grupal, los estudiantes trabajaban en equipos de cuatro o cinco miembros. La puntuación del equipo era la más baja, la suma, o la media de las puntuaciones de sus miembros obtenidos en un test de resultados administrado individualmente después del trabajo en grupo. Todos los miembros del grupo recibian la misma puntuación. En la condición de recompensa individual, los estudiantes recibían sus propias puntuaciones a partir del test, y trabajaban individualmente (Edwards y De Vries, nota 4; Slavin, 1978a, 1978b, nota r) o se les animaba a que trabajasen con otros (De Vries y Mescon, nota 2; De Vries, Mescon y Shackman, nota 3). El número de clases observadas en estos estudios va de 3 a 17 , y el número de alumnos iba de 14 a 242. El nivel de estudios va del tercer grado hasta la enseñanza media, y las materias de estudio incluyen lengua, matemáticas y ciencias sociales.

Los datos acerca del comportamiento de ayuda provienen de las observaciones del trabajo en grupo realizado en cinco estudios (Hanelin, 1978; Johnson, 1979; Slavin, 1978a, 1978b, nota 1 ), y de cuestionarios cumplimentados por estudiantes en tres estudios (De Vries y Mescon, nota 2; De Vries, Mescon y Shackman, nota 3; Edwards y De Vries, nota 4). En los estudios en que se utilizaba la observación de grupos durante su trabajo se definía el comportamiento de ayuda (llamado también tutoria entre compañeros o comportamiento de trabajo entre compañeros) como una interacción entre los miembros del grupo centrado en la tarea. Normalmente los estudiantes eran observados durante intervalos de cinco-seis segundos, de tal manera que los observadores pasaban secuencionalmente de uno a otro miembro del grupo u óbservaban a todos los estudiantes de la clase antes de volver a iniciar el ciclo. Por lo general, la variable de comportamiento era el porcentaje de intervalos empleados en la interacción con los otros centrada en la tarea. En los estudios que utilizaban cuestionarios se pedía a los estudiantes que escribiesen los nombres de los compañeros del grupo (1) a los que ayudaban y (2) de los que recibian ayuda. El número de nombres servía como variable del comportamiento.

Los resultados de los dos estudios que correlacionan el comportamiento de ayuda y los resultados obtenidos en el trabajo no son concordantes. Johnson (1979) informa de una correlación de $.26(\mathrm{p}<.01)$, mientras que Hanelin (1978) informa de correlaciones no significativas $(-.29$ para grupos que trabajan en tareas sencillas, .I 3 para grupos que trabajan en otras más difíciles).

Los otros seis estudios comparan la influencia de las condiciones de recompensa grupal e individual sobre el comportamiento de ayuda y el rendimiento, y ofrecen una evidencia más consistente de relación positiva entre el comportamiento de ayuda y los resultados. Puesto que en un estudio se tuvieron en cuenta dos tipos de contenidos, matemáticas y ciencias sociales (Edwards y De Vries, nota 4), los seis estudios suministran de hecho siete comparaciones de condiciones de recompensa grupal e individual. Entre las siete comparaciones, cinco muestran resultados del comportamiento de ayuda que van en la misma dirección que los 
resultados de rendimiento: cuanto más ayu$\mathrm{da}$, tanto mejor resultado en la condición de recompensa grupal en relación con la condición de recompensa individual (De Vries y Mescon, nota 2; Dé Vries, Mescon y Shackman, nota 3; Edwards y De Vries, nota 4); a igual ayuda, iguales resultados en ambas condiciones (Edwards y De Vries, nota 4; Slavin, nota I). En las otras dos comparaciones (Slavin, I $978 \mathrm{c}, 1978 \mathrm{~b}$ ), la diferencia entre las condiciones y los resultados no responden al comportamiento de ayuda, sino al comportamiento en relación con el trabajo (tiempo empleado trabajando solo o con otros en material relacionado con la tarea). En el estudio de Slavin ( $1978 \mathrm{~b}$ ), por ejemplo, los estudiantes que habían recibido la indicación de que trabajasen con otros se ayudaban más, se centraban menos en la tarea, y alcanzaban unos logros inferiores a los de los estudiantes que recibian la indicación de trabajar por su cuenta. Slavin sugiere que estos resultados permiten cuestionar el rol del comportamiento de ayuda como eslabón entre la cooperación y unos determinados resultados. Sin embargo, una interpretación alternativa posible es que el comportamiento de ayuda produce unos efectos positivos sobre los logros alcanzados que, en el estudio de Slavin, podrian haber quedado empañados por unos efectos negativos, una actitud pasiva o un comportamiento ajeno al trabajo (más adelante volveremos a ello).

Un punto que queda sin aclarar en la investigación citada es si el hecho de ofrecer ayuda y de recibirla tiene una influencia diferente sobre los resultados que se obtienen. Los estudios que vamos a examinar a continuación abordan este punto.

Ofrecer ayuda.-Cinco estudios sobre el aprendizaje de las matemáticas en grupos pequeños distinguen entre ofrecer ayuda y recibirla. Cuatro de ellos se llevaron a cabo en el marco de la clase; el quinto, tuvo lugar en un marco especial, fuera del colegio. Peterson y Janicki (1979) examinaron la participación y los resultados obtenidos por 100 estudiantes de cuarto, quinto y sexto grado en cuatro clases, aprendiendo una unidad sobre fracciones de dos semanas de duración. Peterson, Janicki y Swing (1981) observaron 93 estudiantes de cuarto y quinto grado en dos clases, aprendiendo una unidad de geometria de dos semanas de duración. En el tercer estudio, 48 estudiantes de onceavo grado trabajaron en grupos de cuatro personas durante tres sesiones, para aprender cómo llevar a cabo tres tareas algebraicas y geométricas (Webb, 1980a, 1980c, 1980d; ver también Webb, $1980 \mathrm{ob}$, nota s). Webb (en prensa, a) observó 96 estudiantes de séptimo, octavo y noveno grado en cuatro aulas, mientras trabajaban en una unidad sobre matemáticas del consumidor de una semana de duración. En el último estudio, 77 estudiantes de los grados séptimo y octavo, en dos aulas, aprendieron una unidad de dos semanas de duración sobre exponentes y terminología cientifica (Webb, en prensa, b).

Los datos sobre la interacción del grupo se obtuvieron, en estos estudios, a partir de las observaciones realizadas sobre los grupos durante su trabajo. En los estudios de Peterson, el instrumento de observación exigía al codificador anotar las categorías de comportamiento para cada estudiante y el profesor durante intervalos de veinte segundos consecutivos. También se utilizó un instrumento de observación en el cuarto estudio antes citado (Webb, en prensa a). El observador tomaba notas relativas a la interacción del grupo, identificando al hablante $y$ al receptor de cada intercambio, la categoría de observación, el contenido y la duración del intercambio. En los estudios tercero y quinto, la interacción del grupo se registró mediante un magnetofón y la interacción entre los compañeros se codificó a partir de las transcripciones de las cintas.

Con una sola excepción, los cinco estudios muestran una relación positiva entre el hecho de ofrecer ayuda y los resultados obtenidos. Peterson y Janicki (I979) y Peterson, Janicki y Swing (1981) informan de correlaciones entre .24 y $\quad .29$ $(p<.05)$. Webb (1980a) encuentra que los estudiantes que dan explicaciones de cómo realizar la tarea alcanzan mayores resultados que los estudiantes que no toman parte activa en la interacción del grupo, incluso cuando el nivel de habilidad se mantiene constante. En el estudio de Webb (en prensa, b) también se observa una relación significativa entre el hecho de dar explicaciones y los resultados obtenidos, manteniendo constante la habilidad; la correlación parcial es de .22 
$(p<.05)$. Un nuevo análisis de los datos que aparecian en el estudio de Webb (1980a), distinguiendo entre el material que los estudiantes habian aprendido antes (manipulaciones computacionales y algebraicas) y el material que les resultaba nuevo (un algoritmo para llevar a cabo la tarea matemática) también ofrece resultados positivos (Webb, I98od). La explicación de cómo llevar a cabo manipulaciones computacionales o algebraicas se relaciona con los resultados en esas manipulaciones ( $r$ parcial $=.39, \mathrm{p}<.05)$, y la expresión o descripción del algoritmo se relaciona con el resultado en el algoritmo $(r$ parcial $=.37, \mathrm{p}<.06)$.

La aparición de correlaciones parciales significativas entre las explicaciones dadas $y$ los logros en la habilidad ayudan a clarificar el sentido de la relación entre el fenómeno de ofrecer ayuda y los resultados conseguidos. Hay dos hipótesis acerca de la dirección del efecto: que la interacción influye sobre los resultados obtenidos, y que la interacción depende del nivel de resultados (habilidad). Las correlaciones parciales significativas sirven de base a la primera hipótesis, que la interacción influye sobre los resultados obtenidos.

Recibir ayuda.- Mientras los resultados que relacionan el hecho de ofrecer ayuda con el éxito en el trabajo son directos, los resultados que relacionan el hecho de recibir ayuda con los éxitos conseguidos son más complejos, y sugieren que el hecho de recibir ayuda es una amalgama de diversas variables con diferentes efectos sobre los resultados que se obtienen. Sólo dos de los cinco estudios mencionados informan de una relación significativa entre el hecho de recibir ayuda y los logros observados (Webb, 1980c, ep pren$\mathrm{sa}, \mathrm{b}$; ver también Webb, I980c). Un nuevo y extenso examen de los datos obtenidos en el primer estudio reveló, sin embargo, que la efectividad de la ayuda recibida dependía ( 1 ) de la naturaleza de la ayuda recibida y (2) del comportamiento del estudiante que pedia la ayuda (Webb, $1980 c$ ). Cuando los estudiantes que cometían faltas o que hacian preguntas recibían explicaciones acerca del trabajo, entonces aprendian cómo realizarlo. Si recibian, o bien, el silencio del grupo por respuesta, o sólo soluciones sin explicación alguna, no aprendían a completar la tarea.
Además, los errores cometidos en el test de rendimiento correspondian con el material con el que habian tenido problemas durante el trabajo en grupo. Los resultados del segundo estudio son similares: la relación entre el hecho de recibir explicaciones y los resultados conseguidos es positivo ( $r$ parcial $=.21, \mathrm{p}<.05$ ), mientras que la relación entre el hecho de recibir soluciones sin explicación y los logros alcanzados es negativa ( $r$ parcial $=.44, \mathrm{p}<.01$; Webb, en prensa, b).

Los resultados obtenidos en otro estudio corroboran estos hallazgos, mostrando cómo el hecho de recibir ayuda es efectivo sólo cuando se da como respuesta a la necesidad del alumno (Webb, en prensa, a). La frecuencia en la recepción de ayuda, sin diferenciar entre la ayuda solicitada y la no solicitada, muestra una correlación próxima a cero con los logros alcanzados. Sin embargo, el hecho de recibir ayuda en respuesta a una pregunta, se relaciona de forma significativa con los resultados obtenidos $(r=.19, \mathrm{p}<.05)$.

En un resultado sorprendente, el hecho de no recibir ayuda en respuesta a una pregunta muestra una fuerte relación negativa con los resultados obtenidos en dos de los estudios $(r=-.53, p<.001$, Webb, en prensa, a; y $r=-.55, \mathrm{p}<$. .0or, Webb, en prensa, b). Por tanto, si bien las respuestas de los estudiantes a sus compañeros de equipo puede que no siempre solucionen la confusión, el hecho de no recibir dicha ayuda cuando se necesita parece ir en detrimento de los resultados posteriores.

La importancia del hecho de recibir ayuda cuando se necesita y de recibir respuestas elaboradas cuando son necesarias se ha constatado en un reciente estudio a gran escala realizado con grupos de lectura de primer grado. Aunque el FirstGrade Reading Group Study (Anderson, Everton y Brophy, I979) investigó grupos pequeños dirigidos por un profesor con escaso margen de interacción abierta entre sus componentes, más que grupos pequeños interactivos o cooperativos, sus hallazgos acerca del impacto que las respuestas especificas del profesor al comportamiento de los estudiantes tuvieron sobre los resultados posteriores son paralelos a los arriba descritos. El hecho de recibir un "feedback" terminal a un error -en el cual el profesor da la respuesta correcta, pide a otro compañero que dé 
la respuesta, o permite a otro compañero que dé la respuesta en voz alta- se relaciona negativamente con los resultados posteriores $(\mathrm{P}<. \mathrm{OI})$. El hecho de recibir un "feedback" de proceso a un error ---en el cual el profesor explica cómo obtener la respuesta correcta- se relaciona positivamente con los resultados posteriores $(\mathrm{p}<.0 \mathrm{I})$.

Resumen.- La investigación que relaciona el comportamiento de ofrecer ayuda con los resultados posteriores sugiere que los hechos de ofrecer y recibir ayuda son beneficiosos. Y, lo que es más importante, los hallazgos demuestran que las observaciones del comportamiento de ofrecer ayuda deben estar bien perfiladas: las observaciones deberian distinguir en. tre la ayuda solicitada y la no solicitada, y deberian determinar si se responde a las peticiones de ayuda. Tal como dictaría toda lógica de la instrucción, los estudiantes que necesitasen ayuda, situación indicada por preguntas o errores cometidos, sacarían mayor provecho de las explicaciones que los estudiantes que no necesitasen ayuda, situación que vendría indicada por una ausencia de preguntas y errores (exceptuando a los estudiantes que no participan en absoluto en el trabajo del grupo; ver el próximo apartado). Además, la ayuda que consiste en explicaciones tiene una mayor oportunidad de eliminar la confusión que la ayuda que consiste sólo en dar la respuesta correcta.

El hecho de no distinguir entre diferentes grados de "avuda" y entre ayuda necesitada y no necesitada puede ser el motivo de la falta de resultados significativos en algunos de los estudios mencionados que se limitan a analizar la relación entre la frecuencia del comportamiento de ayuda en general, dentro del grupo y los resultados posteriores (por ejemplo: Hanelin, 1978; Slavin, 1978b). La variable de ayuda no especifica utilizada en dichos estudios ha mezclado, probablemente, acontecimientos positivos y negativos, reduciendo con ello las posibilidades de encontrar una relación sistemática entre interacción y resultados posteriores.

\section{Comportamiento pasivo y no centrado en el trabajo}

Los estudiantes que no se involucran de forma activa en el trabajo del grupo pueden dedicarse, o bien a actividades no relacionadas con el trabajo, o bien a observar pasivamente cómo trabajan los demás. Todos los estudios que han observado comportamientos no centrados en el trabajo muestran una relación negativa, aunque no significativa, entre dichos comportamientos $y$ los resultados posteriores (Hanelin, 1978; Webb, 1980a, en prensa, a). La razón más importante de la ausencia de relación significativa en estos estudios parece ser la limitación en la medida del comportamiento no centrado en el trabajo. Hanelin informa que los estudiantes perdian menos de un 7 por $100 \mathrm{del}$ tiempo del grupo, por término medio, hablando o escuchando a otro compañero en relación a temas que no eran relevantes para el trabajo; $y$ Webb (en prensa, a) observó este comportamiento sólo en el 2 por 100 del tiempo, como término medio. Sin embargo, no resulta claro si el bajo nivel de comportamiento no centrado en el trabajo es una característica del trabajo en grupos pequeños, o bien si es debido a las situaciones en las que tuvieron lugar dichos estudios. Sólo un estudio se realizó en el marco del aula normal (Webb, en prensa, a); los otros estudius se realizaron bajo la dirección de los investigadores, sin que el profesor estuviese presente. Estos últimos estudios puede que hayan subestimado la cantidad de comportamiento no centrado en el trabajo que se observaría en grupos pequeños en el marco natural del aula. Las investigaciones sobre la interacción profesor-estudiante en clase han demostrado la existencia de cantidades considerables de comportamiento no centrado en el trabajo, como se deduce de las observaciones del comportamiento de los niños y de las reprimendas de los profesores por un mal comportamiento, produciéndose una relación negativa entre tales indicadores de comportamiento no centrado en el trabajo y los resultados posteriores (Anderson, Evertson y Brophy, I979; Good y Grouws, 1977; Rosenshine, 1977; Stallings y Kastowitz, 1974). Estos resultados sugieren que la relación negativa encontrada en pequeños grupos, si bien no es significativa, quizá debiera tenerse en cuenta seriamente.

Sólo un estudio investigó la relación existente entre el comportamiento pasivo y los resultados posteriores (Webb, 1980c). El concepto de comportamiento 
pasivo fue definido como ausencia de todo tipo de participación observable en la tarea grupal; no incluía el trabajar individualmente en la tarea. La relación es negativa: la simple observación de la actividad desarrollada por los demás estudiantes durante el trabajo y el hecho de escuchar las explicaciones de los demás no es suficiente para afrender el material. Sin embargo, este estudio era a corto plazo. Las repercusiones a lo largo del tiempo del hecho de adoptar inicialmente en el seno de un grupo una posición pasiva, aún no se han investigado. A medida que estos estudiantes tengan mayor familiaridad con el contexto del grupo puede que participen más en el trabajo grupal.

\section{Participación general}

El único estudio que incluye una medida de participación general, la cantidad de tiempo utilizado en hablar con los restantes miembros del grupo, no encontró relación alguna entre el hecho de hablar y los resultados posteriores (Johnson, I 979). Este resultado no es sorprendente a la luz de los resultados de los estudios ya descritos. El hecho de hablar puede haber implicado la explicación a otros o el hecho de obtener ayuda en respuesta a preguntas, aspectos relacionados positivamente con los resultados posteriores; pero también el hecho de solicitar ayuda y no recibirla o la manifestación de un comportamiento no centrado en el trabajo, aspectos relacionados negativamente con los resultados posteriores. Puede suponerse que estos efectos contrapuestos se neutralizan.

\section{Resumen y discusión}

Las investigaciones que relacionan la interacción en el seno de los grupos con el rendimiento suelen mostrar que el hecho de ofrecer ayuda y el de recibirla se relacionan de manera positiva con los resultados posteriores, y que el comportamiento no centrado en el trabajo y el pasivo se relacionan negativamente con los resultados posteriores. $Y$, de manera no sorprendente, las medidas generales de participación, tales como el número de verbalizaciones, y las variables de ayuda no especificas, tienden a estar débilmente correlacionadas con los resultados poste- riores, o a mostrar relaciones inconsistentes con los mismos.

Aunque las relaciones entre el hecho de dar explicaciones y el de recibirlas $y$ los resultados posteriores tienden a ser positivas, no todos los estudios obtuvieron resultados significativos. Hay dos factores que permiten explicar esos resultados inconsistentes: la complejidad de la tarea y la precisión de las explicaciones. En primer lugar, el hecho de explicar a los demás puede resultar más beneficioso para quien explica cuando el material es complejo, exigiendo integración o reorganización, que cuando el material es simple o directo. En segundo lugar, el hecho de recibir explicaciones acerca de la tarea no tendría por qué estar relacionado con los resultados posteriores, si las explicaciones son imprecisas. Un análisis separado de las explicaciones precisas y de las imprecisas, así como del material complejo y el directo, puede que ayuden a resolver esta cuestión.

El análisis crítico de los diseños $y$ procedimientos utilizados en los estudios aquí expuestos revela que algunas estrategias metodológicas son más informativas que otras para verificar la relación entre la interacción y los resultados posteriores, lo cual sugiere algunas directrices para el diseño de futuros estudios. En primer lugar, las mediciones del comportamiento de cada miembro del grupo son más informativas que los indices de nivel de comportamiento del grupo. La medida generalmente utilizada para el grupo, en estos estudios, fue el comportamiento de ayuda. Sin embargo, no es posible utilizar correlaciones entre la ayuda del grupo y los resultados posteriores para separar los efectos producidos por el hecho de ofrecer ayuda de aquellos producidos por el hecho de recibirla, ni tampoco para explicar la variación de resultados posteriores dentro del grupo. En segundo lugar, las correlaciones entre la interacción y los resultados posteriores en el seno de la misma situación (por ejemplo: la misma estructura de recompensa, instrucciones a todos los grupos para trabajar conjuntamente) suministran una mayor evidencia directa de la relación entre interacción y resultados que las comparaciones entre diferentes tipos de situaciones que varian a lo largo de varias dimensiones (por ejemplo: estructuras de recompensa grupal e individual) y que se prestan a interpretaciones alternativas. 
2. MECANISMOS QUE

RELACIONAN LA

INTERACCION GRUPAL CON

EL RENDIMIENTO

Una importante caracteristica de la que carecen muchas de las investigaciones que relacionan la interacción grupal con los resultados posteriores es la investigación o discusión de cómo la participación en el grupo de trabajo ayuda a sus miembros en el aprendizaje. Los investigadores que han tenido en cuenta este áspecto han elaborado hipótesis de dos tipos de mecanismos que podrian mediar entre la interacción grupal y los resultados posteriores de los estudiantes: los mecanismos que afectan directamente a los procesos cognitivos, y las variables mediadoras pensadas para crear un clima emocional o intelectual favorable para el aprendizaje. Seguidamente tomaremos en consideración ambos mecanismos.

\section{Procesos cognitivos}

Verbalizacion versus reestructuración cognitiva.-Un posible mecanismo que hace de puente entre la interacción grupal y los resultados posteriores hacen referencia a los efectos del mero acto de verbalizar la información. Los estudios del aprendizaje por discriminación, por ejemplo, han demostrado que los estímulos vocalizados se recuerdan más a menudo que los no vocalizados (por ejemplo, Carmean y Weir, 1967; Divesta y Rickards, 1971; Weir y Helgoe, 1968). Además, Gagné y Smith (1962); ver también Davis, 1968) hallaron que la vocalización durante la práctica de una tarea de solución de problemas producía mejores resultados que la no vocalización.

Sin embargo, los resultados de dos estudios recientes ponen en duda la hipótesis de que la simple verbalización del material sea el mecanismo responsable de los mejores resultados obtenidos por los participantes activos en el trabajo en grupo. Durling y Schick (1976) compararon la adquisición de conceptos en tres marcos interactivos: vocalizando a un compañero que también aprende el mismo contenido, vocalizando a un aliado que, supuestamente, también debe aprender dicho contenido, y vocalizando al experimentador que se supone ha dominado ya dicho contenido. Si el mero hecho de verbalizar fuese el mecanismo primario que afectase a los resultados posteriores, entonces las tres condiciones deberían producir niveles de logro similares. En cambio, las tres condiciones produjeron resultados diferentes. En casi todos los criterios de realización, los estudiantes que vocalizaron a un compañero o a un aliado lo hicieron mejor que los estudiantes que vocalizaron al experimentador. Este resultado sugiere que el motivo de la verbalización es más importante para el aprendizaje que el mero acto de dicha verbalización. Como indican Durling y Schick, las personas que vocalizan a un compañero o a un aliado puede que se hayan visto a si mismas como profesores, mientras que los que han vocalizado al experimentador pueden haberse visto a si mismos como estudiantes. La finalidad de la verbalización de quienes se encuentran en un rol de profesor debería ser el ayudar a otra persona a comprender el material, mientras que quienes asumen un rol de estudiante probablemente sólo quieran demostrar el dominio del material.

La evidencia de la eficacia relativa de la verbalización para enseñar y para demostrar el grado del propio aprendizaje viene del segundo estudio reciente: Bargh y Schul (1980). Bargh y Schul compararon el rendimiento obtenido por personas que habian estudiado material verbal para aprendérselo con el obtenido por personas que habian estudiado dicho material para enseñárselo a otro estudiante. Los estudiantes en la condición de profesores mostraron una puntuación más elevada que quienes se encontraban en una situación de no enseñantes. La interpretación que dan Barch y Schul a este resultado puede valer también para los resultados de Durling-Schick. Bargh y Schul sugieren que prepararse para enseñar a alguien puede producir una estructura cognitiva más altamente organizada que el simple hecho de aprender el material para uno mismo. La evidencia de una estructura cognitiva más altamente organizada proviene de la constatación de Bargh y Schul de que las personas que habian estudiado para enseñar mostraban una realización superior tanto en aspectos de detalle periféricos como en aspectos que median el mensaje básico del material. Bargh y Schul (1980) sugieren, además, que no sólo es quien se prepara para enseñar quien reorganiza el material para una presentación más clara, sino también que una persona que esté enseñando activa- 
mente puede reorganizar o clarificar el material sobre la marcha, de modo que ambas cosas permiten al uprofesor ver la cuestion desde nuevas perspectivas, posibilitándole el ver nuevas relaciones, antes impensadas, entre los elementos discretos. Puede que sea esta formación de nuevas relaciones lo que facilite una mejor comprensión fundamental del material» (pág. 595).

La investigación educativa ha adelantado también hipótesis acerca de la importancia de la reestructuración cognitiva en el hecho de recibir ayuda. Gall y Gall (1976) y Slavin (1977) sugieren que el "feedback» del grupo $y$ el hecho de compartir los recursos ayuda a los miembros del grupo a dar nueva forma a sus ideas y a aprender nueva información que quizá no descubriesen por su cuenta. Myers y Lamm (1976) y Wittrock (1974) han subrayado el papel activo que el estudiante desempeña en la reestructuración de dicha información. Myers y Lamm sugieren que la recepción pasiva de la información no es suficiente para que se produzca un cambio en la opinión. Por el contrario, la repetición cognitiva en la cual el individuo procesa, pesa y reformula la información $y$ los argumentos presentados por otros miembros del grupo, resulta necesaria para la interiorización de cambios de actitudes. La noción del individuo como un procesador activo de información, más que un receptor pasivo, constituye también el núcleo del modelo de aprendizaje de Wittrock (1974) como un proceso generativo, que ha sido aplicado al aprendizaje en situaciones grupales (Webb, 1980b). En el modelo de Wittrock, el estudiante genera asociaciones entre la nueva información y los conceptos ya aprendidos. Cuando los estudiantes que trabajan en un mismo grupo se ayudan mutuamente, pueden comparar la nueva información con la información adquirida previamente y modificar o sustituir conceptos ya existentes, según sea necesario.

Las descripciones arriba mencionadas acerca de la reestructuración cognitiva en tanto que mecanismo de enlace entre el hecho de ofrecer o recibir ayuda y los resultados posteriores son ampliamente especulativas. Es necesario que se lleve a cabo una investigación sistemática para comprobar el papel de la reestructuración cognitiva. Esto podría realizarse de varias formas. Un método para obtener datos sobre la reestructuración podría ser el análisis de la interacción verbal. La evidencia de reestructuración en quienes ofrecen ayuda podría incluir la capacidad de decir lo mismo con otras palabras, o reorganizar el material como respuesta a las preguntas, errores u otras indicaciones de confusión de otros miembros del grupo. La evidencia de reestructuración entre quienes reciben ayuda podria incluir la repetición o reorganización de las explicaciones recibidas, aplicando la ayuda recibida a preguntas planteadas por el material, y haciendo otras preguntas acerca de la ayuda recibida. Entonces se podría relacionar la cantidad de comportamiento de reestructuración con los resultados posteriores. Otro método podría ser el recuerdo estimulado en el que los miembros del grupo, al ver un video o escuchar una grabación de la sesión del grupo, tuviesen que describir su proceso mental al ofrecer o recibir ayuda.

Resolución de conflictos.-Mientras Myers y Lamm describen el aprendizaje como requisito previo para que se produzca un cambio de opinión en grupos que tienen que resolver problemas o tomar decisiones, Johnson y Johnson (1979) centran su atención en el papel que juega el cambio de opinión en el aprendizaje. Sugieren un modelo de aprendizaje mediante la resolución de conflictos. En su modelo, la controversia interpersonal lleva a un conflicto conceptual y a sentimientos de inseguridad. Estos sentimientos, a su vez, llevan a los miembros del grupo a buscar información adicional y a revisar las informaciones disponibles desde nuevas perspectivas. Como resultado de buscar nueva información, algunos estudiantes cambian su opinión. Johnson y Johnson (1979) describen las ventajas cognitivas de resolver desacuerdos en los términos siguientes: "Los estudiantes que experimentan un conflicto conceptual como resultado de la controversia están en mejores condiciones para generalizar los principios que aprenden a una más amplia variedad de situaciones que los estudiantes que no experimentan dicho conflicto conceptual» (pág. 67). (Para resultados de investigaciones que apoyan esta interpretación, ver también Anderson y Graesser, 1976; Inagaki y Hatano, 1968, 1977; Kaplan y Miller, 1977; Grimme y Johnson, nota 6). 
Señales verbales y no verbales.-Buckholdt y Wodarski (1978) y Allen y Feldman (1973) han discutido un tipo diferente de mecanismo de proceso cognitivo para explicar la relación existente entre el trabajo en grupo y el rendimiento en el aprendizaje. Este mecanismo concierne las señales verbales y no verbales que utilizan los estudiantes cuando trabajan juntos. Buckhold y Wodarski sugieren que los niños pueden aprender mejor mediante la interacción grupal, porque utilizan un lenguaje que los otros niños pueden entender. Además, los resultados del estudio de Allen y Feldman en la decodificación del comportamiento no verbal sugieren que los niños pueden reconocer mejor y con mayor frecuencia las señales de confusión no verbales de otros niños que los adultos. Allen y Feldman grabaron en vídeo a estudiantes de tercer grado, mientras escuchaban unas lecciones fáciles y difíciles de aritmética. Todos los estudiantes escucharon ambas lecciones. Los videos fueron presentados en un orden al azar, sin sonido, a grupos de alumnos de tercer grado, sexto grado y a profesores adultos con experiencia. Todos los observadores estimaron hasta qué punto cada niño había entendido cada lección. Los resultados demostraron que los estudiantes de tercer y sexto grado eran capaces de diferenciar entre las lecciones fáciles y dificiles, pero no asi los adultos. Los adultos sobreestimaron la comprensión de los niños de la lección difícil. Estos resultados sugieren que los estudiantes que experimentan dificultades mientras aprenden pueden beneficiarse de forma especial trabajando con otros estudiantes.

Allen y Feldman ofrecen dos posibles explicaciones a la mejor habilidad de los niños para decodificar el comportamiento no verbal de otros niños: 1) las señales no verbales de los niños pueden ser diferentes a las de los adultos, y 2) los adultos, al pensar que las señales no verbales de los niños son diferentes de las suyas, pueden interpretar erróneamente el significado de su comportamiento no verbal. Estas hipótesis deberian comprobarse directamente: por ejemplo, pidiendo a niños y a adultos que interpreten señales no verbales especificas emitidas por niños $y$ por adultos.

\section{Variables socioemocionales}

Las variables socioemocionales que ac- túan como mediadores entre los efectos de participación y los resultados posteriores incluyen la motivación, la ansiedad y la satisfacción. Hammond y Goldman (196r) y Slavin (1978c) sostienen que la motivación es una fuerza importante, al menos en las estructuras de recompensa grupal. Establecen como hipótesis la siguiente secuencia de acontecimientos. Cuando cada actuación de un miembro influye en la recompensa del grupo, los miembros de dicho grupo apoyarán los esfuerzos mutuos académicos, lo cual da lugar a un esfuerzo individual mayor. Esta hipótesis necesita ser examinada directamente, utilizando, por ejemplo, cuestionarios o entrevistas que hagan aflorar las percepciones de los estudiantes. También es necesario verificar si el trabajo en grupo aumenta la motivación individual en ausencia de una estructura de recompensa grupal. Las especulaciones realizadas por la dinámica de grupo (por ejemplo, Hackman, 1976; Hackman y Morris, 1975) sugieren que así es.

Las hipótesis de la ansiedad provienen de las observaciones de estudiantes durante el trabajo en grupo y en clases normales. Buckholdt y Wodarski (I978) sugieren que los alumnos de aprendizaje lento pueden aprender más rápidamente de otros compañeros que del profesor, debido a que los niños son más pequeños y despiertan menos miedo que los profesores adultos. Artzt (1979) y Jackson y Riessman (1977) han hecho comentarios similares sobre la mayor disponibilidad de los estudiantes para pedir ayuda a otros compañeros en vez de pedírsela al profesor. El «feedback» de los estudiantes sobre su ansiedad en situaciones diferentes ayudaría a verificar la hipótesis de que los estudiantes experimentan menos ansiedad en grupos pequeños que en otros marcos. Haines y McKeachie (1967) obtuvieron esta información de los alumnos en grandes grupos de discusión (de 20 alumnos cada uno) que aprendian con estructuras de recompensas cooperativas o competitivas. En la condición cooperativa, cada vez que un estudiante contestaba una pregunta correctamente todos los miembros del grupo eran alabados. En la condición competitiva, los estudiantes sólo recibían alabanzas por sus respuestas correctas, y sus puntuaciones eran comparadas con las de los demás niembros del grupo para determinar su nota final. Se pidió a los estudiantes que res- 
pondieran unos cuestionarios acerca de si las condiciones en las que realizaban el aprendizaje promovia una atmósfera fácil y relajada, o si los hacia sentirse ansiosos e incómodos. Las diferencias en los cuestionarios según las condiciones fueron significativas en ambos casos: los estudiantes que trabajaban en una condición cooperativa expresaron menor ansiedad que los estudiantes que lo hacian en condiciones de comperitividad. Sin embargo, los resultados obtenidos por $\mathrm{Hai}$ nes y McKeachie dejan sin respuesta la cuestión de si la interacción con otros o la estructura de recompensa (o ambos factores) ejercen alguna influencia sobre la ansiedad. Serán necesarias otras investigaciones para aclarar estos efectos.

La evidencia directa de satisfacción en grupos cooperativos y competitivos proviene del estudio de Haines y McKeachie (1967) arriba descrito $y$ de un estudio similar dirigido por Crombag ( 1966 ). Contrariamente al estudio de Haines-Mckeachie, Grombag centró su interés en grupos pequeños formados por tres personas. En ambos estudios, los alumnos expresaron mayor satisfacción en la situación cooperativa que en la competitiva. De nuevo queda por verificar empíricamente si el hecho de trabajar en grupos pequeños supone una mayor satisfacción que trabajar en otras situaciones cuando la estructura de recompensa se mantiene constante.

Aunque las especulaciones y los resultados de los estudios arriba citados sugieren que la motivación, la ansiedad y la satisfacción pueden estar relacionadas con los resultados obtenidos en los grupos pequeños, no se ha investigado la relación entre la interacción grupal y dichas variables socioemocionales. Los estudios no hacen referencia a si un aumento de interacción da lugar, por sí solo, a un aumento de la motivación y satisfacción y a una reducción de la ansiedad. Estudios futuros deberian relacionar el nivel de estas variables socioemocionales con la cantidad y tipo de interacción en el grupo, como en el caso de ofrecer y recibir ayuda.

\section{PREDICTORES DE LA INTERACCION}

Los apartados anteriores han mostrado los tipos de interacción verbal que se relacionan con el rendimiento en los grupos pequeños. Algunos tipos de interacción, como el hecho de recibir una explicación detallada en respuesta a preguntas, resultan beneficiosas para los resultados posteriores, mientras que otros, como la discusión no centrada en el trabajo, son negativos para dichos resultados. Estos hallazgos resultan de utilidad limitada para los educadores al no aportar claves sobre cómo diseñar el contexto grupal para promover una interacción beneficiosa y evitar la interacción negativa entre los miembros del grupo. Este apartado centra su atención en las investigaciones que estudian las características de los individuos, las caracteristicas del grupo $y$ las estructuras de recompensa grupal que pueden ayudar a predecir la interacción entre los miembros del grupo. La característica individual que aquí sc cxamina cs la habilidad. Las características del grupo incluyen la composición del grupo en relación con la habilidad y la raza. Se consideran una tras otras todas estas características, así como la estructura de recompensa.

\section{Habilidad del estudiante}

Los cinco estudios mencionados que diferenciaban entre el hecho de ofrecer ayuda $y$ recibirla investigaban también la habilidad del estudiante como elemento que permite predecir la interacción en el grupo. El tipo de habilidad medida en dichos estudios era la habilidad general o la matemática. Cuatro de los cinco estudios muestran que los estudiantes con un elevado nivel de habilidad daban más explicaciones que las que mostraban un nivel de habilidad inferior: las correlaciones entre la habilidad y el hecho de dar explicaciones oscila entre $.24(p<.09) y$ .78 ( $\mathrm{p}<.001$ ) (Peterson y Janicki, 1979; Peterson y otros, 1981; Webb, 1980d, en prensa, b). En cambio, contrastando con estos resultados, sólo un estudio encuentra una relación significativa entre la habilidad y el hecho de recibir explicaciones. Webb (r $980 d$ ) señala que los estudiantes con un nivel de habilidad bajo recibian más explicaciones que los que tenian un nivel superior $(r=-.54$ hasta $-.71, \mathrm{p}<.01)$.

La falta de relación entre la habilidad y el hecho de recibir las explicaciones resulta sorprendente en la mayor parte de los estudios. Un aspecto que todavía no se ha 
abordado, pero que puede que permita explicar este resultado sorprendente, es el hecho de si la interacción en el grupo se predice mejor por la habilidad comparativa dentro de un grupo pequeño o por la habilidad sin referencia a un grupo particular. Ninguno de los cinco estudios examinados midieron la habilidad comparativa en el seno del grupo. Si la habilidad comparativa predice mejor el número de explicaciones que se van a recibir que el nivel de habilidad absoluto, entonces el miembro del grupo menos hảbil deberia ser quien recibiese el mayor número de explicaciones, independientemente de su habilidad absoluta. Cuando los grupos en un estudio varian en su nivel medio de habilidad, o hay fuertes oscilaciones, la correlación entre el nivel absoluto de habilidad y el hecho de recibir explicaciones puede que no sea significativo ni siquiera en el caso de significativo para la habilidad comparativa.

\section{Composición del grupo según el grado de habilidad de sus miembros}

Si bien desde comienzos de siglo se han producido numerosos debates acerca de la agrupación según la habilidad (Espósito, 1973; Goldberg, Passow y Justman, 1966$)$, lo cierto es que se ha prestado poca atención a los procesos que operan en los grupos con márgenes de habilidad diferentes. Los escasos estudios que han comparado la interacción según la composición del grupo sugieren que la mezcla de habilidad es la que dirige los procesos en el grupo. Estos estudios se centraron en el comportamiento de ayuda en el interior del grupo. En un estudio, el comportamiento de ayuda se produjo con frecuencia en grupos heterogéneos y en los homogéneos con una habilidad media, pero no asi en los homogéneos con un nivel elevado de habilidad o con escasa habilidad (Webb, I980a). Otro estudio muestra que los grupos homogéneos con una habilidad media se mostraban más propensos a ayudar que los grupos heterogéneos (Webb, en prensa, b). En un tercer estudio, los grupos heterogéneos produjeron más ayuda que los homogéneos (Webb, en prensa, a). Estos resultados, algo inconsistentes, se pueden explicar en parte por la composición de los grupos heterogéneos en dichos estudios. En los días primeros, los con un elevado nivel de habilidad, uno con un nivel bajo y dos medios. En el tercer estudio, los grupos heterogéneos estaban formados por niveles elevados y medios $o$ bajos y medios; ningún grupo estaba formado por alumnos con niveles altos y bajos. Está claro que es necesario estudiar una variedad de composiciones de grupos según su habilidad antes de poder sacar conclusiones acerca de la relación existente entre la composición del grupo y la interacción. Sin embargo, los resultados sugieren que los efectos de la composición del grupo según su habilidad sobre la interacción dependen del nivel medio de la habilidad del grupo, asi como de la oscilación existente dentro de dicho grupo.

Si bien las comparaciones sistemáticas de la interacción grupal entre grupos con diferente composición son escasas, los psicológos sociales han realizado, a menudo, observaciones informales sobre la interacción en grupos heterogéneos. Sus expectativas en cuanto a los efectos de la heterogeneidad en la habilidad o destrezas específicas de los miembros sobre la interacción tienden a ser pesimistas. Berkowitz (1957) y Mausner (1954), por ejemplo, investigaron la conformidad en parejas heterogéneas y encontraron que los miembros menos adelantados tendían a adaptarse a los juicios de los más avanzados, prescindiendo de la calidad de sus juicios.

Steiner (1972) describe orros dos procesos que operan en los grupos heterogéneos. El primer proceso es el antagonismo, que surge de las dificultades de evaluar y reunir la información. Lo que Steiner considera dificultades "disfuncionales» pueden, sin embargo, resultar beneficiosas para el aprendizaje individual. El modelo de Johnson y Johnson (1979) de resolución de conflictos, por ejemplo, sugiere que los miembros de grupos que experimentan dificultades de este tipo puedan aprender más y obtener unas perspectivas más amplias que los miembros de grupos que no experimentan conflicto alguno.

El segundo mecanismo de Steiner (1972), el descenso en la motivación, es un peligro potencial en todos los grupos de aprendizaje:

"Si se forma un grupo con unos individuos altamente competentes $y$ 
otros claramente menos capaces, los primeros pueden tener la sensación de que deberian recibir una consideración de acuerdo con su mayor nivel de contribución al éxito del grupo. A menos que las recompensas estén distribuidas de manera que reflejen los recursos desiguales de los miembros del grupo, los más competentes pueden renunciar a trabajar a pleno rendimiento. Pueden también sentirse inclinados a disociarse del grupo tan pronto como surja la oportunidad" (pág. I1 2 ).

Si bien el hecho de manipular las estructuras de recompensa tal como sugiere Steiner puede que no siempre produzca el impacto deseado sobre la motivación (ver, por ejemplo, Miller y Hamblin, I 963), puede que sea necesario algún tipo de acción para prevenir la aparición de problemas motivacionales en los grupos heterogéneos.

Es posible que las expectativas de los psicólogos sociales, basadas en general en observaciones de pequeños grupos de discusión en situación no educativas, no sean válidas en el aula y en otros marcos educativos. De hecho, las observaciones anecdóticas realizadas en programas de tutoria entre compañeros $y$ en edades diferentes ofrecen evidencia informal de que existe un antagonismo reducido y un aumento de la motivación en los pares heterogéneos (ver, por ejemplo, el informe de Devin-Sheehan, Feldman y Allen, 1976). Sea como sea, es necesario examinar sistemáticamente estos factores en grupos compuestos por miembros con niveles distintos de habilidad.

Composición racial del grupo.-La bibliografia que examina la composición racial de los grupos, en contraste con lo que ocurre con la que examina otras características grupales o individuales, centra, a menudo, su atención en la interacción dentro del grupo. Estos estudios muestran que en los grupos multirraciales, los estudiantes blancos tienden a mostrarse más activos e influyentes que los estudiantes que pertenecen a minorias (Cohen, 1972; Cohen y Roper, 1972; Cohen y Sharan, nota 7); estos últimos tienden a mostrarse menos seguros y más ansiosos, hablan menos y aportan menos sugerencias $e$ información que los estudiantes blancos (Battle y Rotter, 1963; Delbecq y Kaplan, 1968; Katz, Roberts y Robinson, 1965; Lefcourt y Ladwig, 1965).
Cohen (1972, 1973) atribuye estos resultados a diferencias de "status» entre- los estudiantes blancos y los pertenecientes a minorias.

Sin embargo, la reducida participación de los estudiantes pertenecientes a grupos minoritarios observada en los grupos multirraciales no es inmutable. En una serie de experimentos de campo, Cohen y sus colegas fueron capaces de alterar el modelo de dominio blanco manipulando las expectativas de una realización competente (Cohen, I 973; Cohen y Roper, 1972; Cohen, Lockheed y Lohman, 1976). En estos experimentos, los estudiantes negros recibieron un entrenamiento especial en tareas académicas y no académicas, recibiendo posteriormente las instrucciones de enseñar a estudiantes blancos cómo llevarlas a cabo. A continuación, reunía a todos los estudiantes en grupos multirraciales de un solo sexo (por lo general dos estudiantes negros y dos blancos) para que realizasen una tarea de grupos no relacionada con la que se había utilizado como entrenamiento. El análisis de las grabaciones en vídeo de las sesiones del grupo revelaron niveles iguales de actividad verbal relacionada con la tarea. Este efecto se mantuvo a lo largo de tres semanas de trabajo en el aula realizado en pequeños grupos cooperativos multirraciales. En los casos en los que el tratamiento consistió en entrenar sólo a los estudiantes negros sin manipular al mismo tiempo las expectativas de los estudiantes blancos respecto a las realizaciones de los negros (que es lo que ocurría cuando los estudiantes blancos eran enseñados por los negros), no se alteraba el modelo normal de dominio blanco.

\section{Estructura de recompensa}

Los estudios que examinan los efectos de las estructuras de recompensa sobre el comportamiento del estudiante suelen comparar dos sistemas de recompensa: individual, en la que la puntuación de un miembro, u otro tipo de recompensa, depende de su realización; y grupal, en la que las realizaciones de los miembros se combinan para dar lugar a una puntuación del grupo, de modo que todos los miembros reciben la misma puntuación. La evaluación de los efectos que produce la estructura de recompensa individual o grupal sobre la interacción en el seno del grupo resulta complicado debido a que la 
estructura de recompensa suele confundirse con otros tres factores que afectan al comportamiento del estudiante en el aula. Estos factores son: i) la estructura de la interacción (el hecho de que los estudiantes se vean estimulados o no a trabajar con los demás); 2) la ponderación de las puntuaciones para obtener la pun- tuación global del individuo o del grupo, y 3) el grupo de comparación ( $\mathrm{si}$ la realización del individuo o del grupo se evalúa en relación con, o independientemente de la realización de otros individuos o grupos en la clase). Estos factores aparecen descritos con más detalle en el cuadro I.

\section{CuAdro 1}

Factores con variaciones en los estudios que comparan las estructuras de recompensa individual y grupal

\begin{tabular}{|c|c|}
\hline Facter & Descripción \\
\hline \multicolumn{2}{|l|}{ Estructura de recompensa } \\
\hline Individual & $\begin{array}{l}\text { Los estudiantes reciben puntuaciones de acuerdo con sus } \\
\text { propios resultados. }\end{array}$ \\
\hline Grupal ...... & $\begin{array}{l}\text { La puntuación del grupo se basa en la realización de todos } \\
\text { sus miembros. Todos reciben la misma puntuación. }\end{array}$ \\
\hline \multicolumn{2}{|l|}{ Estructura de interacción } \\
\hline Individual .... & $\begin{array}{l}\text { Los estudiantes trabajan invidualmente, sin interacción con } \\
\text { otros compañeros. Variación: se les permite, pero no } \\
\text { estimula, trabajar con otros compañeros. }\end{array}$ \\
\hline Grupal ............. & $\begin{array}{l}\text { Los estudiantes son distribuidos por grupos y se les } \\
\text { estimula para que interactúen unos con otros. }\end{array}$ \\
\hline \multicolumn{2}{|l|}{$\begin{array}{l}\text { Ponderación de las puntua- } \\
\text { ciones }\end{array}$} \\
\hline $\begin{array}{l}\text { Puntuación grupal no pon- } \\
\text { derada } \ldots \ldots \ldots \ldots \ldots\end{array}$ & $\begin{array}{l}\text { La puntuación del grupo es la media no ponderada de las } \\
\text { puntuaciones obtenidas por sus miembros. }\end{array}$ \\
\hline $\begin{array}{l}\text { Puntuación grupal ponde- } \\
\text { rada } \ldots \ldots \ldots \ldots \ldots\end{array}$ & $\begin{array}{l}\text { La puntuación del grupo es la media ponderada de las } \\
\text { puntuaciones obtenidas por sus miembros, dando mayor } \\
\text { peso a las puntuaciones inferiores. }\end{array}$ \\
\hline $\begin{array}{l}\text { Puntuación individual pon- } \\
\text { derada } \ldots \ldots \ldots \ldots \ldots\end{array}$ & $\begin{array}{l}\text { La puntuación individual es la combinación ponderada de } \\
\text { la puntuación individual y la media del grupo (por } \\
\text { ejemplo, } 33 \text { por } 100 \text {, individual; } 67 \text { por 100, grupo). }\end{array}$ \\
\hline \multicolumn{2}{|l|}{ Grupo de comparación } \\
\hline Toda la clase & $\begin{array}{l}\text { La ejecución del grupo o la individual se evalúa con } \\
\text { relación a la ejecución de toda la clase. }\end{array}$ \\
\hline Categorias de rendimiento & $\begin{array}{l}\text { La ejecución se evalúa con relación a estratos homogéneos } \\
\text { dentro de la clase. }\end{array}$ \\
\hline Uno mismo. & $\begin{array}{l}\text { La ejecución se evalúa con relación a las ejecuciones previas } \\
\text { individuales o grupales. }\end{array}$ \\
\hline
\end{tabular}

Tres de los factores que aparecen en el cuadro i fueron examinados explícitamen- estructura de recompensa, la ponderación de las puntuaciones y el grupo de comparación. El otro factor, la estructura de la 
interacción, suele ser un factor "escondido", y/o bien se confunde con la estructura de recompensa, o bien se mantiene constante. En pocos estudios se reconoce la estructura de la interacción como un factor por derecho propio, variando de forma sistemática. Los estudios que aquí se revisan están agrupados en tres categorías según el tratamiento que se da en sus diseños a la estructura de la interacción: 1) la estructura de recompensa es confundida con la estructura de la interacción; 2) la restructura de la interacción se mantiene constante, y 3) la estructura de recompensa se cruza con la de interacción.

La estructura de recompensa se confunde con la de la interacción.-Los estudios con el primer diseño incorporan la estructura de la interacción a la de la recompensa. En la condición de recompcnsa grupal los estudiantes suelen trabajar en grupos y reciben una puntuación de grupo. El elemento clave de la condición de recompensa del grupo es, en estos estudios, el equipo. Los alumnos son distribuidos en equipos de cuatro o cinco miembros para que practiquen con material académico presentado con anterioridad por el maestro. Tras practicar en equipos, los estudiantes demuestran su aprendizaje en torneos (como en los Teams-Games-Tournaments, TGT; ver Slavin, I977) o en preguntas (como en los Student Teams and Achievement Divisions, STAD; ver Slavin, 1978b). La puntuación de un equipo es la combinación de las puntuaciones obtenidas por sus miembros en el torneo o en las preguntas.

En la condición de recompensa individual, los estudiantes suelen practicar individualmente con el material académico y reciben puntuaciones individuales basadas en su propio trabajo. En muchos estudios, a los alumnos. se les permite trabajar con otros compañeros durante las sesiones de práctica, pero no son estimulados a hacerlo. En otros estudios, a los alumnos no se les permite trabajar con otros compañeros.

Muchos de los estudios que comparan recompensa grupal, trabajo en grupo con recompensa individual-trabajo individual, encuentran que los estudiantes en las condiciones de grupo muestran una frecuencia más elevada de tutoría entre compañeros, comportamiento centrado en el trabajo y participación total que los estudiantes en régimen individual (Slavin,
I 977, I 978a, nota I; De Vries y Edwards, 1973; De Vries, Edwards y Wells, nota 8, nota 9).

Sin embargo, algunos estudios encuentran que la estructura de recompensa interacciona con otros dos factores. Uno es la ponderación diferencial de las puntuaciones obtenidas por los compañeros de equipo. DeVries, Edwards y Wells (nota 8 , nota 9) y Edwards y DeVries (nota 4) introdujeron la ponderación diferencial en sus condiciones de recompensa de equipo. En una condición, la puntuación del equipo era la puntuación media no ponderada de las obtenidas por sus miembros. En la otra condición, las puntuaciones de los compañeros de equipo se ponderaron inversamente al rango, de manera que las puntuaciones bajas eran más fuertemente ponderadas. Esta fuerte ponderación de las puntuaciones más bajas iba dirigida a impulsar a los estudiantes a ayudar a los miembros más flojos del equipo. Edwards y DeVries (nota 4) introdujeron una tercera condición con el mismo sistema de ponderación de la segunda, pero que no era competitiva, en el sentido de que la información semanal de la clase no incluia el ranking entre equipos, y éstos sólo eran comparados con sus realizaciones anteriores. Los tres estudios ofrecieron diferentes resultados. DeVries, Edwards y Wells (nota 9) observaron mayor ayuda en la condición ponderada que en la no ponderada; DeVries, Edwards y Wells (nota 8) no hallaron diferencias en el comportamiento entre las condiciones ponderadas y las no ponderadas; y Edwards y DeVries (nota 4) hallaron una relación compleja entre la estructura de recompensa y el sistema de ponderación. Los estudiantes con recompensa de equipo y con ponderación igual fueron quienes más se ayudaron; los estudiantes con recompensa de equipo, ponderación diferencial y condición no competitiva, fueron quienes menos se ayudaron; y la frecuencia de ayuda en el caso de recompensa de equipo, ponderación diferéncial, y condición competitiva y condiciones de recompensa individual ocupaba un lugar intermedio.

El otro factor añadido al diseño básico (recompensa grupal - trabajo en grupo vs. recompensa individual- trabajo individual) fue el grupo de comparación, grupo con relación al cual se evalúa la realización de un estudiante. Slavin 
(i $978 \mathrm{a}$, nota 1) utilizó un diseño factorial para separar los efectos de la estructura de recompensa y del grupo de comparación. La estructura de recompensa (equipo vs. individual) se cruzó con el grupo de comparación (comparación con toda la clase vs. compatáción con los iguales). En el caso del comportamiento centrado en el trabajo, Slavin informó de los principales efectos significativos de la estructura de recompensa y del grupo de comparación: los estudiantes que trabajaban en la condición de equipo se centraban más en el trabajo que cuando estaban en condición individual, y los estudiantes que se hallaban en la condición de comparación con iguales se centraban más en el trabajo que los que se hallaban en una condición de comparación con toda la clase. Sin embargo, en lo que hace referencia al comportamiento de ayuda, existía una interacción entre la estructura de recompensa y el grupo de comparación. Mientras, los estudiantes en la condición de equipo se ayudaban más entre sí que quienes trabajaban en una condición individual, los efectos del grupo de comparación dependían de la condición de recompensa. En el caso de recompensa grupal, la frecuencia de sparición del comportamiento de ayuda era, aproximadamente, igual en la comparación entre iguales que en la condición de comparación con toda la clase. Sin embargo, en caso de recompensa individual, los estudiantes que estaban en la condición de comparación con toda la clase se ayudaban más entre sí que quienes se encontraban en. la condición de comparación con iguales.

Slavin (1978a) describió la razón de la baja frecuencia de aparición del comportamiento de ayuda en la combinación de comparación con igual recompensa individual en los términos siguientes: «Puesto que los estudiantes no sabian con qué compañeros iban a ser comparados, posiblemente eran reticentes a ayudar a nadie por temor a que su propio lugar en la clasificación pudiese verse afectado negativamente" (pág. 539). Tales especulaciones podrian ser fácilmente confirmadas en estudios futuros mediante cuestionarios a cumplimentar por los estudiantes.

La estructura de la interación se mantiene constante.-Mientras los estudios que se engloban en la primera categoría exami- tructura de recompensa y de la interacción, cuatro estudios variaron la estructura de recompensa mientras mantenían constante la de la interacción.

Se pidió a los estudiantes que trabajaban en una estructura de recompensa individual y a los que lo hacian en una estructura de recompensa grupal que trabajasen en grupo. Un estudio utilizó combinaciones complejas de recompensas individuales y de grupales; en todas las condiciones se propició el que trabajasen con otros (Wodarski, Hamblin, Buckholdt y Ferritor, 1973; ver también Buckholdt y Wodarski, 1978). Las cuatro condiciones de recompensa fueron: 1) 100 por 100 individual, en la que a los estudiantes se les recompensaba por su propia realización; 2) 67 por 100 individual, 33 por 100 grupal, en la que el 67 por 100 de la recompensa a un estudiante se basaba en su realización y el 33 por 100 restante en la media de las cuatro notas más bajas del grupo; 3 ) 33 por roo individual, 67 por Ioo grupal, en la que el 33 por Ioo de la recompensa al estudiante se basaba en su realización y el 67 por roo en la media de las cuatro notas más bajas del grupo; y 4) 100 por 100 grupal, en la que a los estudiantes se les recompensaba sólo en base a las cuatro notas más bajas del grupo.

Los cuatro estudios muestran unos resultados similares. Los estudiantes que se encontraban en la condición de recompensa de grupal se ayudaron más entre sí que quienes se encontraban en la condición de recompensa individual (De Vries y Edwards, 1974; De Vries y Mescon, nota 2; De Vries, Mescon y Shackman, nota 3). En Wodarski y otros, a medida que aumentaba la proporción de la recompensa grupal aumentaba también la frecuencia del comportamiento de ayuda. Como afirma Slavin en su trabajo (1978a), los estudiantes que se encontraban en una condición de recompensa individual al I00 por 100 mostraron una baja incidencia de tutoría, incluso en los casos en los que se les animaba a ayudarse mutuamente.

Estos resultados no son sorprendentes. Como dice Slavin, los estudiantes que estaban en una condición individual se hacian la competencia mutuamente frente a la recompensa individual, con lo que no estaban motivados para ayudarse mutuamente. Era de esperar que las instrucciones de trabajar juntos no lograsen 
superar la tendencia de los estudiantes a ignorarse mutuamente $\mathrm{y}$, a obstruirse los unos a los otros en el camino, tendencias que suelen adscribirse a aquellos individuos que se hallan en una situación competitiva (ver, por ejemplo, Davidson, Agreen y Davis, I978; Johnson y Johnson, 1974).

La estructura de recompensa se cruza con la de interaccion.-Comparativamente con los nueve estudios que, o bien confundian la estructura de recompensa con la de interacción, o bien mantenian constante la estructura de interacción, sólo un estudio separó los efectos de la estructura de recompensa de los de la estructura de interacción. Slavin (i978c) comparó el comportamiento de los estudiantes en cuatro condiciones: r) recompensa grupal con tutoria entre compañeros; 2) recompensa grupal sin tutoría entre compañeros; 3) recompensa individual con tutoría entre compañeros, y 4) recompensa individual sin tutoria entre compañeros. La condición recompensa grupal-tutoría entre compañeros se parece a las condiciones de grupo de los anteriores estudios. En la condición de recompensa grupal-no tutoría, los estudiantes se sentaban en grupos, pero recibian instrucciones de trabajar individualmente. En la condición de recompensa individual-condición de tutoria, los estudiantes eran estimulados para que trabajasen con otros estudiantes, pero recibian la recompensa de acuerdo con su propia realización. En la condición de recompensa individual-no tutoria los estudiantes trabajaban solos. Slavin examinó dos variables de comportamiento: la frecuencia del comportamiento centrado en el trabajo y la frecuencia del comportamiento de ayuda. Como afirmaba Slavin en su hipótesis, los estudiantes que trabajaban en condiciones de recompensa grupal se centraban en el trabajo por periodos de tiempo significativamente más largos que quienes se encontraban en una condición de recompensa individual. Sin embargo, contrariamente a lo que esperaba Slavin, los estudiantes que estaban en condiciones de no tutoría se centraban en el trabajo por períodos de tiempo más largos que quienes se hallaban en condiciones de tutoría. Por lo que respecta a la variable de ayuda, los estudiantes que se hallaban en las condiciones de recompensa grupal se ayudaban más que quienes se encontraban en condicio- nes de recompensa individual. Aunque Slavin no comparó el comportamiento de ayuda en las condiciones de tutoria $y$ de no tutoria, tal información resultaría útil para confirmar la efectividad de las instrucciones dadas a los sujetos en el sentido de que trabajasen con los compañeros.

Los resultados de Slavin (1978b, 1978c) sugieren que la recompensa grupal es una fuerza más motivante para promover la cooperación entre los estudiantes que el hecho de favorecer el trabajo con los compañeros. Unicamente instrucciones a los estudiantes para que trabajen con los demás no producirá demasiado comportamiento cooperativo si el hecho de ayudar a un compañero no afecta la nota que uno vaya a obtener (o cualquier otro tipo de refuerzo), o si el hecho de ayudarlo se interpreta como un aumento de las posibilidades de recibir una nota más baja o una recompensa menor.

Estructura de recompensa $y$ productividad del grupo.-Todos los estudios que examinan la estructura de recompensa tienen una característica común. El rendimiento de cada individuo contribuye a su recompensa, independientemente de la estructura de recompensa. Contrariamente a lo que ocurre con estos estudios, la investiguación dirigida por Deutsch (1949, I960a, 1960b), frecuentemente citada en las revisiones de las estructuras de recompensa, se centraba no en el rendimiento individual, sino en la productividad del grupo. Puesto que las leyes que rigen la interacción en los grupos en los que se intenta aumentar la comprensión mutua pueden diferir de las que rigen la interacción en los grupos en los que se intenta obtener la mejor solución a la tarea, la interacción resultante en los estudios de Deutsch fue diferente a la de los estudios revisados más arriba.

Deutsch (1 949, I 960a, I 960b) comparó la interacción dentro del aula en grupos cooperativos y competitivos que habian recibido las instrucciones de resolver acertijos y problemas de relación humana. En la condición cooperativa se juzgaba a los distintos grupos de acuerdo con calidad y cantidad de ideas producidas para cada problema. Todos los miembros del grupo recibian la misma calificación. En la condición competitiva, cada miembro era calificado de acuerdo con la calidad y cantidad de ideas aportadas en relación 
con las de los demás miembros del grupo. Coincidiendo con los resultados obtenidos en los estudios citados más arriba, Deutsch halló que los estudiantes que trabajaban en grupos cooperativos trabajaban más juntos, se mostraban más cooperativos y participaban de forma más equivalente que los que trabajaban en grupos competitivos. Sin embargo, contrariamente a lo que ocurre en los otros estudios, los estudiantes que trabajaban en grupos cooperativos mostraban una mayor división del trabajo, para evitar la duplicación de esfuerzos, que los estudiantes que lo hacian en grupos competitivos. En palabras de Deutsch (1960a), "(La división del trabajo) permitía a los miembros dividir el trabajo en sus diferentes aspectos, posibilitando a los diferentes miembros trabajar simultáneamente en esos componentes» (pág. 436).

Aunque la divisióa del trabajo fue una fórmula eficaz para que los grupos de Deutsch completasen su tarea, esta estratcgia puede resultar contraproducente para el aprendizaje. Una persona que sistemáticamente realice el mismo aspecto de la tarea puede que no llegue a aprender cómo llevar a cabo otros aspectos de la misma. Si una estructura de recompensa basada en el rendimiento estimula la participación de todos los miembros del grupo en todos los aspectos de la tarea, es de esperar que el aprendizaje sea mayor que el alcanzado con una estructura de recompensa basada en la calidad del producto del grupo. Todavia está pendiente un estudio sistemático de esta cuestión.

Resumen: El resultado de los estudios en los que se examina el impacto de la estructura de recompensa sobre la interacción en el grupo sugiere que recompensar a un estudiante por el rendimiento alcanzado por los otros, además del suyo propio, promueve la cooperación y consigue que todos los miembros presten mayor atención al trabajo. Por otra parte, la estructura de recompensa parece ejercer un mayor impacto sobre ia interacción en el grupo que las instrucciones que buscan influir directamente en la interacción entre los miembros del grupo. Sin embargo, estas conclusiones pueden verse suavizadas por los hallazgos de interacciones entre la estructura de recompensa, el sistema de ponderación y el grupo de comparación. Cuando se tienen en cuenta la ponderación de las puntuaciones y el grupo de comparación, las condiciones de recompensa del grupo no siempre producen la cantidad mas importante de comportamiento de ayuda. Para que los factores que influyen sobre la interacción dejasen de ser ambiguos sería necesario manipular de forma sistemática, o al menos establecer de forma explicita, las instrucciones de interacción, la ponderación de las puntuaciones y cl grupo de compara ción.

\section{Resumen de los predictores de la interacción}

La interacción entre los estudiantes se puede predecir en parte a partir de las caracteristicas del individuo, del grupo y de la estructura de recompensa. De todos los predictores que hemos examinado, la habilidad y la estructura de recompensa son los que mantienen una relación más consistente con la interacción entre los estudiantes. Los estudiantes con un nivel de habilidad mayor dieron más explicaciones que quienes tenían un nivel inferior. Si se recompensaba a los estudiantes por los resultados obtenidos por todos los miembros del grupo se lograba promover, de forma regular, el comportamiento de ayuda. Las instrucciones dadas a los alumnos para que trabajasen con sus compañeros no siempre resultaba efectiva, a menos que fuesen acompañadas por una recompensa grupal. Los pocos estudios que examinan la composición del grupo en cuanto al grado de habilidad de sus miembros hallaron efectos significativos de interacción, pero puesto que utilizaban diferentes sistemas para formular los grupos no se pueden sacar conclusiones generales. Finalmente, los grupos multirraciales tendian a inhibir la participación de los estudiantes pertenecientes a minorías, si bien este efecto se podía superar manipulando las expectativas de los alumnos con relación a la competencia de cada cual. Para investigaciones futuras, la predicción de la interacción partiendo simultáneamente de diferentes características individuales y de grupo puede resultar una estrategia analitica más productiva para relacionar los inputs con la interacción.

\section{DISCUSION GENERAL}

La investigación sobre el papel que 
juega la interacción entre los estudiantes como predictor del rendimiento o como un resultado en si mismo sugiere que dicha interacción puede ser un eslabón importante entre las caracteristicas del individuo, del grupo, de la situación y dei rendimiento. Sin embargo, como hemos visto, los estudios de los que se disponen hasta el momento no logran formar un cuerpo de investigación de cohesión son: 1) metodológicas, incluyendo los diseños no comparables, la ausencia de procedimientos detallados o apropiados de observación, unidades de observación inapropiadas y estrategias de análisis simplistas, y 2) sustantivas, incluyendo medidas de interacción entre estudiantes excesivamente generales e inapropiadas. Vamos a considerar brevemente estos problemas, así como otros aspectos importantes y tener en cuenta en investigaciones futuras.

\section{Aspectos metodológicos y sugerencias}

Comparabilidad de los diseños. - Uno de los mayores obstác:ılos para llegar a sacar conclusiones generales acerca del impacto de la interacción entre estudiantes sobre el rendimiento y del impacto de las características de input sobre la interacción, es la marcada diferencia existente entre los diseños de las investigaciones revisadas. Todos los estudios difieren en, por lo menos, uno de los factores siguientes, y a menudo en cuatro o más: nivel (elemental, medio, superior), materia a estudiar (ciencias, ciencias sociales, lenguas, lectura, matemáticas -incluyendo el álgebra, la geometria, las fracciones, computación, probabilidad-), tamaño de los grupos (de dos hasta cinco miembros), regla de composición de los grupos (habilidad, raza, homogéneos, heterogéneos, azar, ninguna regla), estructura de recompensa (cooperación intragrupal, competencia intergrupal, competencia intragrupal, individualista), instrucciones para la interacción entre los miembros del grupo (propiciada, permitida, no permitida) y duración del estudio (de una semana hasta un año). Además, muchos estudios no mencionan estos factores, dejando al lector inseguro respecto al diseño. Los investigadores que hallan resultados contradictorios con investigaciones previas buscan a menudo excusas señalando diferencias en uno o más de los factores que acabamos de indicar, como, por ejemplo, el nivel elegido o la materia a aprender.
Estas excusas no bastan; deben verificarse empiricamente los efectos de dichos factores sobre la interacción en los grupos. Por lo menos se debería explicitar las condiciones con la suficiente claridad como para permitir las comparaciones entre los estudios y servir de guia a las réplicas.

Procedimientos de observación.-Una de las mayores dificultades en la evaluación de los resultados de los estudios en los que se ha investigado la interacción grupal es la falta de descripciones detalladas de los procedimientos de observación. Muchos informes se limitan a hacer una lista de las variables de observación. Otros presentan definiciones y ejemplos o citan instrumentos de observación existentes. Sin embargo, pocos suministran detalles suficientes para determinar la comparabilidad de los procedimicntos de observación y de las variables entre estudios o para guiar las réplicas.

Otro problema concierne la adecuación de los procedimientos de observación, incluso en aquellos casos en los que se especifican con detalle. Un procedimiento común de observación es el sistema de recogida rotativa de muestras, en el que los estudiantes son observados secuencialmente durante intervalos de tres a treinta segundos. En la mayoría de los casos, los intervalos de observación para cada estudiante es demasiado corto para recoger las secuencias de intercambios entre estudiantes, o entre estudiante y profesor, o para obtener suficiente información acerca del contenido de dichos intercambios. Así, los datos sobre comportamiento interactivo consisten en "flashes" periódicos de interacción verbal más que en informes continuos de interacción. Esta limitación tiene implicaciones importantes respecto a la relevancia de las variables de observación utilizadas en la mayor parte de los estudios. Un resultado importante al que se llega en varios estudios es que el rendimiento no depende de acontecimientos aislados, sino de secuencias de interacción, incluyendo, por ejemplo, las respuestas a las preguntas o errores de los estudiantes. Unos procedimientos de observación que captasen mayor riqueza de detalles podrian ser los registros narrativos o la grabación mediante cinta magnetofónica o vídeo.

Varios estudios utilizan cuestionarios, en lugar de observaciones independien- 
tes, para obtener informes de los estudiantes acerca de sus propios roles y de los de otros compañeros en la interacción en el grupo. Los informes elaborados por los propios estudiantes no pueden sustituir las observaciones de la interacción en el grupo por dos razones. En primer lugar, aunque estos informes pueden aportar una información global acerca de la interacción, como la identificación de los «ayudantes» en el seno del grupo, no pueden suministrar detalles de intercambios especificos entre los estudiantes. En segundo lugar, no se ha investigado la precisión de los informes de los estudiantes acerca de la interacción y su correspondencia con las observaciones independientes.

Si bien, los informes realizados por los propios estudiantes no pueden sustituir las observaciones independientes, pueden aportar informaciones valiosas sobre cómo evalúan los estudiantes la interacción en el grupo (por ejemplo, si consideran que las explicaciones son comprensibles o útiles) y sobre los elementos que facilitan $o$ inhiben la interacción. Las observaciones pueden mostrar que algunos estudiantes participan muy poco, pero puede que no revelen las razones de esta falta de participación. Los informes dè los propios alumnos, por otro lado, pueden facilitar claves sobre la variedad de elementos que influyen en su conducta, incluyendo, por ejemplo, la valoración de su habilidad o estatus dentro del grupo, características personales de otros miembros del grupo, normas del grupo e influencias sobre la motivación relacionadas o no con el grupo. Utilizados conjuntamente, las observaciones y los informes realizados por los propios alumnos pueden suministrar una imagen completa de la interacción en grupos pequeños.

Unidad de observación.-Un procedimiento común utilizado en las observaciones de la interacción grupal es el registro de la frecuencia con la que aparece un determinado comportamiento (por ejemplo, el de ayuda) en un grupo, sin ligar dicha información a un miembro especifico del mismo. Sin embargo, la información a nivel de grupo tiene una utilidad limitada para predecir o comprender el impacto de la experiencia del grupo sobre el rendimiento de sus miembros. Por ejemplo, una elevada frecuencia de ayuda en un grupo puede que no resulte beneficioso para el rendimiento de todos los miembros del grupo si las explicaciones no se dirigen a quienes más las necesitan. Por otra parte, incluso una elevada correlación entre la frecuencia del comportamiento de ayuda en un grupo y el rendimiento no dice nada acerca de los efectos que produce el recibir ayuda por un lado y el ofrecerla por otro.

Lo que resulta interesante es que muchos estudios sí comienzan por obtener datos acerca del comportamiento de los estudiantes por separado, como en el sistema de muestreo rotatorio descrito antes, pero luego los combinan en índices grupales para su análisis. El mantenimiento de los datos relativos al comportamiento individual de los estudiantes a lo largo de todo el análisis puede suministrar una información más rica y más precisa sobre el impacto que las experiencias grupales de los alumnos ejercen sobre su rendimiento.

Estrategias analiticas.-Los estudios futuros que aspiren a predecir la interacción de los estudiantes a partir de las caracteristicas de los individuos, del grupo y de la situación deberian tener en cuenta las complejas relaciones existentes entre estos factores. La mayor parte de los investigadores han estudiado cada característica por separado. Las características de los individuos, grupos y situación raramente han sido examinadas en el mismo estudio. Las características individuales pueden ser susceptibles de malas interpretaciones cuando se aislan de las características del grupo. Las investigaciones realizadas acerca de la habilidad individual y la composición del grupo en cuanto a habilidad sugieren que los estudiantes con un nivel de habilidad bajo, por ejemplo, pueden tener experiencias diferentes en grupos heterogéneos y homogéneos. En los grupos heterogéneos los estudiantes con un bajo nivel de habilidad tienden a recibir explicaciones de estudiantes con un nivel de habilidad superior, mientras que en los grupos homogéneos puede que no reciban explicación alguna. La imagen seguramente se complicará cuando se introduzcan varias estructuras de recompensa. Todavía no se sabe, por ejemplo, cómo pueden interaccionar los efectos de la composición del grupo en cuanto a habilidad y las estructuras de recompensa. Quizá las recompensas grupales, que tienden a promover el comportamiento de 
ayuda, equilibren la tendencia a la desaparición de la ayuda en algunos grupos con un nivel de habilidad homogénea. Puede ser que los resultados que hemos descrito deban reinterpretarse cuando se tengan en cuenta simultáneamente otras variables.

\section{Aspectos sustantivos y sugerencias}

Variables de observacion.-El problema de las variables de observación excesivamente generales oscurece la interpretación de las relaciones entre la interacción de los estudiantes y las variables de input y el rendimiento. En este informe hemos reiterado más de una vez la importancia de examinar categorías específicas de interacción entre estudiantes en lugar de medidas generales como el número de verbalizaciones o el número de segundos empleados en hablar. Las investigaciones resumidas sugieren que algunas variables de interacción se relacionan positivamente con el rendimiento alcanzado (por ejemplo, el hecho de ofrecer ayuda o recibirla), otras se relacionan negativamente (por ejemplo, el comportamiento no centrado en el trabajo, el comportamiento pasivo), mientras que otras puede que no se relacionen en absoluto con el rendimiento. Los análisis que no logren distinguir entre estas variables seguramente no producirán resultados sólidos ni consistentes.

Interpretación psicologica.-Deben perfilarse interpretaciones psicológicas de las relaciones input-proceso-resultado. El hecho de cómo la interacción grupal promueve el aprendizaje todavía no se comprende bien ni se han formulado demasiadas hipótesis al respecto. Se podrian investigar explicitamente las variables que unen la interacción con el rendimiento y las características de input con la interacción. La evidencia sobre los procesos cognitivos, como la reestructuración que se produce cuando los alumnos reciben $u$ ofrecen ayuda, puede obtenerse a partir del análisis de las explicaciones y de las respuestas a las explicaciones (por ejemplo, la reverbalización o reorganización del material, al dar ejemplos o descripciones elaboradas), o estimulando procesos cognitivos mediante la utilización de vídeos o grabaciones en magnetofón de la interacción grupal. Los informes elaborados por los propios alumnos pueden suministrar informaciones relativas a va- riables socioemocionales como motivación, ansiedad y satisfacción, que actúan tal vez mediadores entre los efectos de la participación y el rendimiento alcanzado.

Además, la investigación debe clarificar qué mecanismos operan sobre el grupo como un todo y cuáles están ligados a experiencias especificas de la interacción grupal. Si, por ejemplo, el aumento de la moral de los estudiantes juega un papel importante en la mejora del rendimiento, entonces los individuos pueden sacar provecho de la experiencia grupal independientemente de su propio nivel de participación. Si el aumento de la comprensión, más que de la moral, es el responsable de un mejor rendimiento, entonces la participación de cada estudiante en el grupo se hace necesaria. Lo más probable es que haya muchos factores que influyan simultáneamente sobre las relaciones entre input-interacción-resultados.

Estabilidad de la interacción.-Los estudios longitudinales de la interacción grupal deben determinar cómo cambian las experiencias de los estudiantes a lo largo del tiempo. Aunque muchos estudios han observado la interacción en el aula a lo largo de varios meses, ningún investigador ha estudiado los cambios que se producen en los modelos de interacción.

La abundante y variada bibliografia sobre la evolución de los grupos sugiere que los procesos grupales pasan por una serie de estadios a medida que los miembros del grupo van ganando familiaridad tanto entre ellos como con la tarea (ver, por ejemplo, Bales, 1950; Bennis y Shepard, 1956; Bion, ig6r; Mills, 1964; Schutz, 1958; Tuckman, 1965). Todavía no se ha estudiado si los mismos modelos de evolución pueden caracterizar a los grupos en situaciones educativos, como tampoco el impacto de tales cambios sobre el rendimiento.

En resumen, la evidencia aportada por los estudios sobre la interacción de los estudiantes en grupos pequeños no es, por el momento, lo suficientemente consistente como para autorizar la conclusión poco cualificada de que el análisis de las modalidades de interacción resolverá todas las discrepancias existentes entre los estudios que investigan el aprendizaje en grupos cooperativos. Pero la evidencia es lo suficientemente fuerte como para defender la importancia de la interacción 
para el aprendizaje en grupos. Además, puede resultar altamente fructífero considerar las caracteristicas del input del indi- viduo, del grupo y de la situación, la interacción en el grupo y el rendimiento como un sistema de relaciones.

\section{Notas}

1 SI.AVIN, R. E.: Student learning teams and scores adjusted for past achievement: $A$ summary of field experiments. (Report No. 227). Center for Social Organization of Schools. The Johns Hopkins University, 1977.

2 DEVRIES, D. L., y MESCON, I. T..: Teams-games-lournament: An effective task and reward siructure in the elementary grades. (Report No. 189). Center for Social Organization of Schools, The Johns Hopkins University, I 975 .

3 DE VRIES; D. L.; MESCON, 1. T.; yे SHACKMAN. S. L.: Teams-games-tournament in the elemintary classroom. A replication. (Report No. 190). Center for School Organization of Schools. The Johns Hopkins University, 1975 .

4 EDWARDS, K. J., y DEVRIES, D.: The effects of teams-games-tournoment and two instructional variations on classroom process, student attitudes, and student achievement. (Report No. 172). Center for Social Organization of Schools, The Johns Hopkins University, 1974.

5 WEBB, N. M.: Learning in individual and small group settings. (Tech. Report No. 7). Stanford, Calif: Aptitude Research Project, School of Education, Stanford University, 1977.

6 GRIMME, N., y JOHNSON, D. W.: The effects of controversy on students' motivation and achievement. Unpublished manucript. University of Minnesota, 1978.

7 COHEN, E. G., y SHARAN, S.: Modifying status relations among Israeli youth. Manuscript submitted for publication, 1979 .

8 DEVRIES, D. L.; EDWARDS, K. J., y WELls, E. H.: Teams-games-fournament in the social studies classroom: Effects on academic acbievements, student attitudes, cognitive beliefs, and classroom climate. (Report 1973). Center for Social Organization of Schools. The Johns Hopkins University, 1974.

9 DEVRIES, D. L.; EDWARDS, K. J., y WelLS, E. H.: Team competition effects on classroom group process. (Report No. 174): Center for Social Organization of Schools, The Johns Hopkins University, 1974

\section{Resumen}

Si bien en los últimos años ba aumentado considerablemente el mumero de investigaciones acerca del aprendizaje en grupos pequeños en régimen de cooperación, pocos estudios se ban centrado en los procesos de interacción que tienen lugar dentro de los grupos. Este trabajo se centra en el papel de la experiencia de los estudiantes en interacción en grupos pequeños durante el aprendizaje. Se examina la investigacion que bace referencia a los tres aspectos del aprendizaje en grupos pequeños: i) la relacion entre interacción y resultado, 2) proceso cognitivo y mecanismos socioemocionales que unen la interacción y los resultados y 3) caracteristicas del individuo, del grupo y de la estructura de recompensa que predicen la interacción en grupos pequeños. Se discuten aspectos metodológicos y sustantivos para evaluar e integrar los resultados de investigaciones previas, y como directrices para futuras investigaciones. La conclusión es que el rol de un individuo en la interaccion en el grupo ejerce una importante influencia sobre el aprendizaje, y que la interacción se puede predecir a partir de las caracteristicas muiltiples del individuo, del grupo y de la situación marco.

\section{Summary}

While research on learning in cooperative small groups bas greatly increased during the past several years, few studies bave focused on the interaction processes occurring within groups. This review focuses on the role of the student's experience in small group interaction in learning. Research bearing on three aspects of small group learning is examined: 1) the relationship between interaction and acbievement, 2) cognitive process and social-emotional mechanisms bridging interaction and acbievement, and 3) characteristics of the individual, growp, and reward structure that predict interaction in small groups. Metbodological and substantive issues are discussed to evaluate and integrate research findings, and as guidelines for further research. The conclusion is that an individual's role in group interaction is an important influence on learning, and that interaction can best be predicted from multiple characteristics of the individual, group, and setting.

\section{Résumé}


le róle de Pexpérience qui ont les itudiants en interaction dans des petits groups pendant la realisation de tâches d'apprentissage. On passe en revue les recherches qui etudient trois aspects de l'apprentissage en petits groups: 1) la relation qui existe entre Pintéraction et les résultats de Papprentissage; 2) les processus cognitifs et les mécanismes socio-émotivanels qui relient lintéraction et les résultats de lapprentissage, et 3) les caractéristiques de l'individu, du groupe et de la structure de recompense qui permettent de predire linteraction dans les petits groups. On discute quelques questions méthodologiques et théoriques afin d'integrer les résultats de ces recherches et de formuler quelques suggestions pour les recherches futures. La conclusion est que le róle de l'individu dans fintéraction groupale a une très grande importance pour rapprentissage et que Ion peut prédire l'interaction a partir de sertaines caractéristiques de Pindivids, du groupe et de la situation.

\section{Referencias}

ALleN, V. L., y FELdman, R. S.: «Learning through tutoring: Low-achieving children as tutors». Journal of Experimental Education, 1973, 42, 1-5.

ANDERSON, L. M.; EverTSON, C. M., y BROPHY, J. E.: "An experimental study of effective teaching in first-grade reading groups". The Elementery Scbool Journal, 1979, 79, 193-273.

ANDERSON, N. H., y GRAESSER, C.: "An information integration analysis of attitude change in group discussion». Journal of. Personality and Social Psychology, 1976, 34, $210-222$.

ARTzT, A.: "Student teams in mathematics class". Mathematics Teacher, 1979, 72, $505-908$.

BALES, R. F.: Interaction process analysis: $A$ metbod for the study of small groups. Chicago: The University of Chicago Press, igso.

BARgh, J. A., y SChUL, Y.: "On the cognitive benefits of teachingi. Jowrnat of Edutational Psychology, 1980, $72,593-604$.

BAtTle, E. S., y ROTTER, J. B.: “Children's feelings of personal control as related to social class and ethnic group». Journal of Personality, 1963, 3I, 482-490.

BENNis, W. G., y SHEPARD, A.: “A theory of group development». Human Relations, 1956, 9, $419-437$.

BERKOWITZ, L.: "Effects of perceived dependency relationships upon conformity to group expectations". Journal of Abnormal and Social Psycbology, 1957, 5 , $350-394$.

BıoN, W. R.: Experiences in groups: And other papers, Nueva York: Basic Books, 1961.

BUCKHOLDT, D. R., y WODARSKI, J. S.: "The effects of different reinforcement systems or cooperative behaviors exhibited by children in classroom contexts». Journal of Research and Development in Education, $1978,12,50-68$.

CARmean, S. L., y WeIR, M. W.: «Effects of verbalizations on discrimination learning and retention». Journal of Verbal Learning and Verbal Behavior, $1967,6,549-590$.

COHEN, E. G.: «Interracial interaction disability». Human Relations, 1972, 25 , 9-24.

COHEN, E. G.: «Modifying the effects of social structure». American Behavioral Scientist, 1973, 16, $861-879$.

COHEN, E. G.; LOCKHEED, M. E., y LOHMAN, M. R.: "The center for interracial cooperation: A field experiment". Sociology of Education, .1976, 49, 47-58.

COHEN, E. G., y ROPER, S.: "Modification of interracial interaction disability: An application of status characteristics theorym. American Sociological Review, 1972, 37, 643-697.

Crombag, H. F.: "Cooperation and competition in means-interdependent triads: A replication». Journal of Personality and Social Psychology, 1966, 4, 692-695.

DAVIDSON, N.; AGREEN, L., y DAVIS, C.: "Small group learning in junior high school mathematics". School Science and Mathematics, $1978,21,23-30$.

DAvis, J. H.: "Verbalization, experimenter presence, and problem solving". Journal of Personality and Social Psychology, 1968, 8, 299-302.

DEI.BECQ, A. L., y KAPI,AN, S. J.: "The myth of the indigenous community leader within the war on poverty". Academy of Management Journal, $1968, I I, 11-29$.

DEUTSCH, M.: «An experimental study of the effects of cooperation and competition upon group process». Human Relations, 1949, 2, 199-231.

DEUTSCH, M.: "The effect of motivational orientation upon trust and suspicion". Human Relations, 1960, 13, $123-139$ (a).

DEUTSCH, M.: "The effects of cooperations and competition upon group process". En D. Cartwright y A. Zander (F.ds.), Group dynamics: Research and theory (2nd. ed.). Nueva York: Row Peterson \& Co., $1960(\mathrm{~b})$.

Devin-Sheehan, L.; Feldoman, R. S., y AlLen, V. L.: «Research on children tutoring children: A critical review". Retien of Educational Research, 1976, 46, 359-385.

DEVRIES, D. L., y EDWARDS, K. J.: «Learning games and student teams: The effects on classroom process". American Educational Research Journal, 1973, 10, 307-318.

DEVRIES, D. L., y EDWARDS, K. J.: "Student teams and learning games. Their effects on cross-race and cross-sex interaction». Journal of Educational Psychology, 1974, 66, 741-749.

DiVesta, F. J., y RiCKARDS, J. P.: "Effects of labeling and articulation on the attainment of concrete, abstrat, and number conceptsm. Journal of Experimental Psychology, 1971, 88, 41-49.

DURI.ING, R., y SCHICK, C.: «Concept attainment by pairs and individuals as a function of vocalization". Journal of Education Pyscbology, 1976, 68, 83-91.

ESPOSITO, D.: «Homogeneous and heretogeneous grouping: Principal findings and implications for evaluating and designing more effective educational environments". Review of Educational Research, 1973, 43, $163-179$.

GAGNE, R. M., y SMITH, E. C., Jr.: "A study of the effects of verbalization on problem solving". Journal of Experimental Psychology, 1962, 63, 12-18. 
Gall, M. D., y Gall, J. P.: "The discussion method». In N. L. Gage (Ed.). The psychology of teaching memods. Chicago: The University of Chicago Press, 1976.

Goldberg, M. L.; Passow, A. H., y Justman, J.: The effects of ability grouping. Nueva York. Teaching College Press, 1966.

GoOD, T. L., y Grouws, D. A.: "Teaching effectiveness in fourth-grade mathematics classrooms". In G. D. Borich \& K. S. Fenton (Eds.). The appraisal of teaching: Concepts and process. Reading, Mass.: Addison-Wesley, 1977.

HaCKman, J. R.: «Group influences on individuals». En M. D. Dunnett (Ed.). Handbook of industrial and organizational psycbology. Chicago: Rand McNally College Publishing, 1976.

HACKMAN, J. R., y MORRIS, C.: «Group tasks, group interaction process, and group performance effectivenes: A review and proposed integrationm. En L. Berkowitz (Ed.). Advances in experimental social psychology (vol. 8), 197s.

HAINES, D. B., y MCKEACHIE, W. J.: "Cooperation versus competitive discussion methods in teaching introductory psychologym. Journal of Educational Psycbology, 1967, 88, 386-390.

HAMMOND, L. K., y GOLDMAN, M.: "Competition and non-competition and its relationship to individual and group productivityn. Sociometry, 1961, 24, 46-60.

HANELIN, S. J.: Learning, behavior and attitudes under individual and group contingencies. Tesis doctoral médita. Universidad de California, Los Angeles, 1978.

INAGAKI, K., y HATANO, G.: “Motivacional influences en epistemic observation». Japanese Journal of Educational Psychology, 1968, 16, $221-228$.

INAGAKI, K., y HATANO, G.: "Amplication of cognitive motivation and its effects on epistemic observation". American Educational Research Journal, 1977, 14,48 5-491.

Jackson, V. C., y Riessman, F.: «A children teaching children program». Theory into Practice, 1977, 16, 280-284.

JoHNsoN, J. A.: "Learning in peer tutoring interactions: The influence of status role change, time-on-task, feedback, and verbalizationn. Tesis doctoral, Universidad de California, Los Angeles, 1978). Dissertation Abstracts International, 1979, 39, \$469a-\$470a. (University Microfilms No. 79-06, 175.)

JOHNSON, D. W.: "Student-student interaction: The neglected variable in education». Educational Researcher, $1981,10(1), 3-10$.

Johnson, D. W., y Johnson, R. T.: «Instructional goal structure: Cooperative competitive, or individualisticm. Review of Educational Research, 1974, 44, 312-340.

JOHNSON, D. W., y JOHNSON, R. T.: "Conflict in the classroom: Controversy and learning". Reviey of Educational Research, 1979, 49, \1-70.

KAPLAN, M. F., y MilleR, C.: "Judgments and group discussion: Effect of presentation and memory factors on polarizationm. Sociometry, 1977, 40,337-343.

KATZ, I.; ROBERTS, S. P., y ROBINSON, J. M.: «Effects of difficulty, race of administrator, and instructions on Negro digit-symbol performance». Journal of Personality and Social Psycbology, 1963, 2, 33-59.

LEFCOURT, H. M., y LADWIG, G. W.: "The effect of reference groups upon Negroes' task persistence in a biracial competitive gamen. Journal of Personality and Social Psychology, 1969, 1 , 668-671.

MAUSNER, B.: "The effect of one partner's success in a relevant task on the interaction of observer pairs". Journal of Abnormal and Social Psychology, 1954, 49, 557-560.

MICHAELS, J. W.: “Classroom reward structures and academic performance». Revien of Educational Research, $1977,47,87-98$.

Miller, L. K., y Hamblin, R. L.: "Interdependence, differential rewarding and productivity". American Sociological Revien, 1968, 28, 768-778.

MiLls, T. M.: Group transformation. Englewood Cliffs, N. J. Prentice-Hall, 1964.

MYers, D. G., y LAMm, H.: "The group polarization phenomenon». Psycbological Bulletin, 1976, 83, 602-627.

Peterson, P. L., y JANiCKI, T. C.: uIndividual characteristics and children's learning in large-group and small-group approaches". Journal of Educational Psychology, 1979, 71, 677-687.

Peterson, P. L.; Janicki, T. C., y Swing, S. R.: "Ability x treatment interaction effects on children's learning in large-group and small-group approaches». American Educational Research Journal, 1981, 18 , 453-473.

ROSENSHINE, B.: «Review of teaching variables and student achievement». En G. D. Borich \& K. S. Fenton (Eds.), The appraisal of teacbing: Concepts and process. Reading, Mass.: Addison-Wesley, 1977.

SCHUTZ, W. C.: FIRO: A three-dimensional theory of interpersonal bebavior. Nueva York: Holt, Rinehart, 1938.

SHARAN, S.: "Cooperative leaming in small groups: Recent methods and effects on achievement, attitudes and ethnic relationsm. Revien of Educational Research, 1980, 10, 24 1-272.

SLAVIN, R. E.: "Classroom reward structure: An analytical and practical review". Revies of Educational Research, 1977, 47, 633-650.

SLAVIN, R. E.: "Student teams and comparison among equals: Effects on academic performance and student attitudesn. Journal of Educational Psychology, 1978, $70,532-538$ (a).

St.AviN, R. E.: "Effects of student teams and peer tutoring on academic achievement and time ontask". Journal of Experimental Education, 1978, 48, 292-297 (b).

SLAvin, R. E.: "Students teams and achievement division». Journal of Research and Development in Education, $1978,12,39-49$ (c).

SLAVIN, R. E.: "Cooperative learning". Revien of Educational Research, 1980, 50, 315-342 (a).

SLAVIN, R. E.: "Cooperative learning in teams: State of the art". Educational Psychologist, 1980, 15, 93-1 11 (b).

Stal.lings, J. A., y KaSKowitz, D.: Follow through classroom evaluation 1972-1973. Menio Park, Calif.: Stanford Research Institute, 1974.

STEINER, I. D.: Group process and productivity. Nueva York: Academic Press, 1972.

Tuckman, B. W.: «Developmental sequence in small groups". Psychological Bulletin, $1969,63,384-399$.

WEBB, N. M.: «Group process: The key to learning in groupsw. New Directions for Methodology of Social and Behavioral Science: Issues in Aggregation, 1980, 6, 77-87 (a). 
WisB, N. M.: "Group process and learning in an interacting group". The Quarterly Newletter of the Laboratory of Comparative Human Cognition, 1980, 2, 10-1s (b).

WEBB, N. M.: "A process-outcome analysis of learning in group and individual settings». Educational Psychologist, 1980, $15,69-83$ (c).

WEBB, N. M.: "An analysis of group interaction and mathematical errors in heterogeneous ability groups". British Journal of Educational Psycbology, 1980, 30, 1-11 (d).

WEBB, N. M.: "Group composition, group interaction and achievement in cooperative small groups". Journal of Educational Psychology, en prensa, a.

WEBB, N. M.: "Peer interaction and learning in cooperative small groups». Journal of Educational Psychology, en prensa, $b$.

WEIR, M. W., y HeIGOE, R. S.: "Vocalization during discrimination: Effects of a mixture of two types of verbalization patterns". Journal of Verbal Learning and Verbal Behavior, 1968, 7, 842-844.

WITTROCK, M. C.: “Learning as a generative process". Educational Psychologist, 1974, 11, 87-95.

WODARSKI, ]. S.; HAMBLIN, R. L.; BuCKhOLDT, D. R., y FERRITOR, D. E.: «Individual consequences versus different shared consequences contingent on the performance of low-achieving group members». Journal of Applied Social Psychology, 1973, 3, 276-290. 